\title{
A precise measurement of the partial decay width ratio $R_{\mathrm{b}}{ }^{0}=\Gamma_{\mathrm{b} \overline{\mathrm{b}}} / \Gamma_{\text {had }}$
}

DELPHI Collaboration

\begin{abstract}
The partial decay width of the $\mathrm{Z}$ to $\mathrm{b} \overline{\mathrm{b}}$ quark pairs has been measured by the DELPHI detector at LEP using data taken in the years 1992 to 1995. Decays of b-hadrons were tagged by several methods using tracks with large impact parameters and/or reconstructed secondary vertices, complemented by event shape variables. Combining these methods in a multivariate analysis the value

$$
\frac{\Gamma(Z \rightarrow b \bar{b})}{\Gamma(Z \rightarrow h a d)}=0.21634 \pm 0.00067(\text { stat }) \pm 0.00060(\text { syst })
$$

was obtained, where the c $\bar{c}$ production fraction was fixed to its Standard Model value.
\end{abstract}


P.Abreu ${ }^{21}, \quad$ W.Adam ${ }^{50}, \quad$ T.Adye ${ }^{36}, \quad$ P.Adzic ${ }^{11}, \quad$ Z.Albrecht ${ }^{17}, \quad$ T.Aldeweireld ${ }^{2}, \quad$ G.D.Alekseev ${ }^{16}, \quad$ R.Alemany ${ }^{49}$, T.Allmendinger ${ }^{17}$, P.P.Allport ${ }^{22}$, S.Almehed ${ }^{24}$, U.Amaldi ${ }^{9}$, S.Amato ${ }^{47}$, E.G.Anassontzis ${ }^{3}$, P.Andersson ${ }^{44}$, A.Andreazza ${ }^{9}$, S.Andringa ${ }^{21}$, P.Antilogus ${ }^{25}$, W-D.Apel ${ }^{17}$, Y.Arnoud ${ }^{14}$, B.Åsman ${ }^{44}$, J-E.Augustin ${ }^{25}$, A.Augustinus ${ }^{9}, \quad$ P.Baillon ${ }^{9}$, P.Bambade $^{19}$, F.Barao ${ }^{21}$, G.Barbiellini ${ }^{46}$, R.Barbier ${ }^{25}$, D.Y.Bardin ${ }^{16}$, G.Barker ${ }^{9}$, A.Baroncelli ${ }^{38}$, M.Battaglia ${ }^{15}$, M.Baubillier ${ }^{23}$, K-H.Becks ${ }^{52}$, M.Begalli ${ }^{6}$, P.Beilliere ${ }^{8}$, Yu.Belokopytov ${ }^{9,53}$, K.Belous ${ }^{42}$, A.C.Benvenuti ${ }^{5}$, C.Berat ${ }^{14}$, M.Berggren $^{25}$, D.Bertini2 ${ }^{25}$, D.Bertrand ${ }^{2}$, M.Besancon ${ }^{39}$, F.Bianchi ${ }^{45}$, M.Bigi ${ }^{45}$, M.S.Bilenky ${ }^{16}$, M-A.Bizouard ${ }^{19}$, D.Bloch $^{10}$, H.M.Blom ${ }^{30}$, M.Bonesini2 ${ }^{2}$, W.Bonivento ${ }^{27}$, M.Boonekamp ${ }^{39}$, P.S.L.Booth ${ }^{22}$, A.W.Borgland ${ }^{4}$, G.Borisov ${ }^{19}$, C.Bosio ${ }^{41}$, O.Botner ${ }^{48}$, E.Boudinov ${ }^{30}$, B.Bouquet ${ }^{19}$, C.Bourdarios ${ }^{19}$, T.J.V.Bowcock ${ }^{22}$, I.Boyko ${ }^{16}$, I.Bozovic ${ }^{11}$, M.Bozzo ${ }^{13}$, P.Branchini ${ }^{38}$, T.Brenke ${ }^{52}$, R.A.Brenner ${ }^{48}, \quad$ P.Bruckman ${ }^{18}, \quad$ J-M.Brunet ${ }^{8}, \quad$ L.Bugge $^{32}, \quad$ T.Buran ${ }^{32}$, T.Burgsmueller ${ }^{52}$, P.Buschmann ${ }^{52}$, S.Cabrera ${ }^{49}$, M.Caccia $^{27}, \quad$ M.Calvi ${ }^{27}$, A.J.Camacho Rozas ${ }^{40}, \quad$ T.Camporesi ${ }^{9}$, V.Canale ${ }^{37}$, F.Carena ${ }^{9}$, L.Carroll ${ }^{22}$, C.Caso ${ }^{13}$, M.V.Castillo Gimenez ${ }^{49}$, A.Cattai ${ }^{9}$, F.R.Cavallo ${ }^{5}$, V.Chabaud ${ }^{9}$, M.Chapkin ${ }^{42}$, Ph.Charpentier ${ }^{9}, \quad$ L.Chaussard ${ }^{25}, \quad$ P.Checchia $^{35}, \quad$ G.A.Chelkov ${ }^{16}, \quad$ R.Chierici ${ }^{45}$, P.Chliapnikov ${ }^{42}$, P.Chochula ${ }^{7}$, V.Chorowicz ${ }^{25}$, J.Chudoba ${ }^{29}$, P.Collins ${ }^{9}$, R.Contri ${ }^{13}$, E.Cortina ${ }^{49}$, G.Cosme ${ }^{19}$, F.Cossutti ${ }^{39}$, J-H.Cowell ${ }^{22}$, H.B.Crawley ${ }^{1}$, D.Crennell ${ }^{36}, \quad$ G.Crosetti ${ }^{13}, \quad$ J.Cuevas Maestro ${ }^{33}, \quad$ S.Czellar $^{15}, \quad$ G.Damgaard ${ }^{28}, \quad$ M.Davenport $^{9}$, W.Da Silva ${ }^{23}, \quad$ A.Deghorain ${ }^{2}, \quad$ G.Della Ricca ${ }^{46}, \quad$ P.Delpierre $^{26}, \quad$ N.Demaria $^{9}, \quad$ A.De Angelis ${ }^{9}, \quad$ W.De Boer $^{17}$, S.De Brabandere ${ }^{2}$, C.De Clercq ${ }^{2}$, B.De Lotto ${ }^{46}$, A.De Min ${ }^{35}$, L.De Paula ${ }^{47}$, H.Dijkstra ${ }^{9}$, L.Di Ciaccio ${ }^{37}$, J.Dolbeau ${ }^{8}$, K.Doroba ${ }^{51}$, M.Dracos ${ }^{10}$, J.Drees ${ }^{52}$, M.Dris ${ }^{31}$, A.Duperrin' ${ }^{25}$, J-D.Durand ${ }^{25,9}$, G.Eigen ${ }^{4}$, T.Ekelof ${ }^{48}$, G.Ekspong ${ }^{44}$, M.Ellert ${ }^{48}$, M.Elsing ${ }^{9}$, J-P.Engel ${ }^{10}$, B.Erzen ${ }^{43}, \quad$ M.Espirito Santo ${ }^{21}$, E.Falk ${ }^{24}$, G.Fanourakis ${ }^{11}$, D.Fassouliotis ${ }^{11}$, J.Fayot $^{23}$, M.Feindt ${ }^{17}$, A.Fenyuk ${ }^{42}$, P.Ferrari ${ }^{27}$, A.Ferrer ${ }^{49}$, E.Ferrer-Ribas ${ }^{19}$, S.Fichet ${ }^{23}$, A.Firestone ${ }^{1}$, P.-A.Fischer ${ }^{9}$, U.Flagmeyer $^{52}$, H.Foeth ${ }^{9}$, E.Fokitis ${ }^{31}$, F.Fontanelli ${ }^{13}$, B.Franek ${ }^{36}$, A.G.Frodesen ${ }^{4}$, R.Fruhwirth ${ }^{50}$, F.Fulda-Quenzer ${ }^{19}$, J.Fuster $^{49}$, A.Galloni ${ }^{22}$, D.Gamba ${ }^{45}$, S.Gamblin ${ }^{19}$, M.Gandelman $^{47}$, C.Garcia ${ }^{49}$, J.Garcia ${ }^{40}$, C.Gaspar $^{9}$, M.Gaspar $^{47}$, U.Gasparini $^{35}$, Ph.Gavillet ${ }^{9}$, E.N.Gazis ${ }^{31}$, D.Gele ${ }^{10}$, N.Ghodbane ${ }^{25}$, I.Gil ${ }^{49}$, F.Glege ${ }^{52}$, R.Gokieli ${ }^{51}$, B.Golob ${ }^{43}$, G.Gomez-Ceballos $^{40}$, P.Goncalves ${ }^{21}$, I.Gonzalez Caballero ${ }^{40}, \quad$ G.Gopal ${ }^{36}, \quad$ L.Gorn ${ }^{1,54}$, M.Gorski ${ }^{51}$, Yu.Gouz ${ }^{42}$, V.Gracco ${ }^{13}$, J.Grahl ${ }^{1}$, E.Graziani ${ }^{38}$, C.Green ${ }^{22}$, H-J.Grimm ${ }^{17}$, P.Gris ${ }^{39}$, K.Grzelak ${ }^{51}$, M.Gunther ${ }^{48}$, J.Guy ${ }^{36}$, F.Hahn ${ }^{9}$, S.Hahn $^{52}$, S.Haider ${ }^{9}$, A.Hallgren ${ }^{48}$, K.Hamacher ${ }^{52}$, F.J.Harris ${ }^{34}$, V.Hedberg ${ }^{24}$, S.Heising ${ }^{17}$, J.J.Hernandez ${ }^{49}$, P.Herquet ${ }^{2}$, H.Herr ${ }^{9}$, T.L.Hessing ${ }^{34}$, J.-M.Heuser ${ }^{52}$, E.Higon ${ }^{49}$, S-O.Holmgren ${ }^{44}$, P.J.Holt ${ }^{34}$, D.Holthuizen ${ }^{30}$, S.Hoorelbeke ${ }^{2}$, M.Houlden ${ }^{22}$, K.Huet ${ }^{2}$, G.J.Hughes ${ }^{22}$, K.Hultqvist ${ }^{44}$, J.N.Jackson ${ }^{22}$, R.Jacobsson ${ }^{9}$, P.Jalocha ${ }^{9}$, R.Janik ${ }^{7}$, Ch.Jarlskog $^{24}$, G.Jarlskog $^{24}$, P.Jarry ${ }^{39}$ ， B.Jean-Marie ${ }^{19}$, E.K.Johansson ${ }^{44}$, P.Jonsson ${ }^{25}$ ， C.Joram ${ }^{9}$, P.Juillot ${ }^{10}$, F.Kapusta ${ }^{23}$, K.Karafasoulis ${ }^{11}$, S.Katsanevas ${ }^{25}$, E.C.Katsoufis ${ }^{31}$, R.Keranen ${ }^{17}$, B.P.Kersevan ${ }^{43}$, B.A.Khomenko ${ }^{16}$, N.N.Khovanski ${ }^{16}$, A.Kiiskinen ${ }^{15}$, B.King ${ }^{22}$, A.Kinvig ${ }^{22}$, N.J.Kjaer ${ }^{30}$, O.Klapp ${ }^{52}$, H.Klein ${ }^{9}$, P.Kluit ${ }^{30}$, P.Kokkinias ${ }^{11}$, M.Koratzinos ${ }^{9}$, V.Kostioukhine ${ }^{42}$, C.Kourkoumelis ${ }^{3}$, O.Kouznetsov ${ }^{16}, \quad$ M.Krammer $^{50}, \quad$ C.Kreuter ${ }^{9}, \quad$ E.Kriznic $^{43}, \quad$ J.Krstic $^{11}$, Z.Krumstein $^{16}$, P.Kubinec ${ }^{7}$, W.Kucewicz ${ }^{18}$, J.Kurowska ${ }^{51}$, K.Kurvinen ${ }^{15}$, J.W.Lamsa ${ }^{1}$, D.W.Lane ${ }^{1}$, P.Langefeld ${ }^{52}$, V.Lapin ${ }^{42}$, J-P.Laugier ${ }^{39}$, R.Lauhakangas ${ }^{15}$, G.Leder ${ }^{50}$, F.Ledroit ${ }^{14}$, V.Lefebure ${ }^{2}$, L.Leinonen ${ }^{44}$, A.Leisos ${ }^{11}$, R.Leitner $^{29}$, G.Lenzen $^{52}$, V.Lepeltier ${ }^{19}$, T.Lesiak ${ }^{18}$, M.Lethuillier ${ }^{39}$, J.Libby ${ }^{34}$, D.Liko ${ }^{9}$, A.Lipniacka ${ }^{44}$, I.Lippi ${ }^{35}$, B.Loerstad ${ }^{24}$, J.G.Loken ${ }^{34}$, J.H.Lopes ${ }^{47}$, J.M.Lopez ${ }^{40}$, R.Lopez-Fernandez ${ }^{14}$, D.Loukas ${ }^{11}$, P.Lutz ${ }^{39}$, L.Lyons ${ }^{34}$, J.MacNaughton ${ }^{50}$,

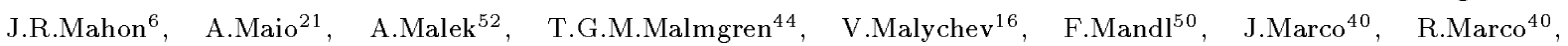
B.Marechal $^{47}, \quad$ M.Margoni $^{35}, \quad$ J-C.Marin ${ }^{9}, \quad$ C.Mariotti ${ }^{9}, \quad$ A.Markou ${ }^{11}, \quad$ C.Martinez-Rivero ${ }^{19}, \quad$ F.Martinez-Vidal ${ }^{49}$, S.Marti i Garcia ${ }^{9}$, N.Mastroyiannopoulos ${ }^{11}$, F.Matorras ${ }^{40}$, C.Matteuzzi ${ }^{27}$, G.Matthiae ${ }^{37}$, J.Mazik ${ }^{29}$, F.Mazzucato ${ }^{35}$, M.Mazzucato $^{35}$, M.Mc Cubbin ${ }^{22}$, R.Mc Kay ${ }^{1}$, R.Mc Nulty ${ }^{22}$, G.Mc Pherson ${ }^{22}$, C.Meroni ${ }^{27}$, W.T.Meyer ${ }^{1}$, E.Migliore ${ }^{45}$, L.Mirabito $^{25}$, W.A.Mitaroff ${ }^{50}$, U.Mjoernmark ${ }^{24}$, T.Moa ${ }^{44}$, M.Moch $^{17}, \quad$ R.Moeller ${ }^{28}$, K.Moenig ${ }^{9}$, M.R.Monge ${ }^{13}$, X.Moreau $^{23}$, P.Morettini ${ }^{13}$, G.Morton ${ }^{34}$, U.Mueller ${ }^{52}$, K.Muenich ${ }^{52}$, M.Mulders ${ }^{30}$, C.Mulet-Marquis ${ }^{14}$, R.Muresan ${ }^{24}$, W.J.Murray ${ }^{36}$, B.Muryn ${ }^{14,18}$, G.Myatt ${ }^{34}$, T.Myklebust ${ }^{32}$, F.Naraghi ${ }^{14}$, F.L.Navarria ${ }^{5}$, S.Navas ${ }^{49}$, K.Nawrocki ${ }^{51}$, P.Negri $^{27}$, N.Neufeld ${ }^{9}, \quad$ N.Neumeister ${ }^{50}, \quad$ R.Nicolaidou ${ }^{14}, \quad$ B.S.Nielsen ${ }^{28}, \quad$ M.Nikolenko ${ }^{10,16}, \quad$ V.Nomokonov ${ }^{15}$, A.Normand $^{22}$, A.Nygren ${ }^{24}$, V.Obraztsov ${ }^{42}$, A.G.Olshevski ${ }^{16}$, A.Onofre ${ }^{21}$, R.Orava ${ }^{15}$, G.Orazi ${ }^{10}$, K.Osterberg ${ }^{15}$, A.Ouraou $^{39}$, M.Paganoni2 ${ }^{27}$, S.Paiano ${ }^{5}$, R.Pain ${ }^{23}$, R.Paiva ${ }^{21}$, J.Palacios ${ }^{34}$, H.Palka ${ }^{18}$, Th.D.Papadopoulou ${ }^{31}$,

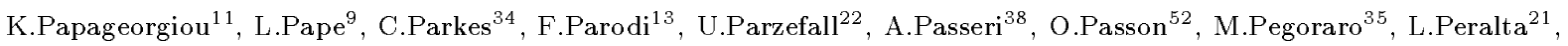
M.Pernicka ${ }^{50}$, A.Perrotta ${ }^{5}$, C.Petridou ${ }^{46}$, A.Petrolini ${ }^{13}$, H.T.Phillips ${ }^{36}$, F.Pierre ${ }^{39}$, M.Pimenta ${ }^{21}$, E.Piotto ${ }^{27}$, T.Podobnik $^{43}$, M.E.Pol ${ }^{6}$, G.Polok ${ }^{18}$, P.Poropat ${ }^{46}, \quad$ V.Pozdniakov $^{16}, \quad$ P.Privitera $^{37}, \quad$ N.Pukhaeva ${ }^{16}, \quad$ A.Pullia ${ }^{27}$, D.Radojicic ${ }^{34}$, S.Ragazzi ${ }^{27}$, H.Rahmani ${ }^{31}$, D.Rakoczy ${ }^{50}$, P.N.Ratoff ${ }^{20}$, A.L.Read ${ }^{32}$, P.Rebecchi ${ }^{9}$, N.G.Redaelli ${ }^{27}$, M.Regler ${ }^{50}$, D.Reid ${ }^{9}$, R.Reinhardt ${ }^{52}$, P.B.Renton ${ }^{34}$, L.K.Resvanis ${ }^{3}$, F.Richard ${ }^{19}$, J.Ridky ${ }^{12}$, G.Rinaudo ${ }^{45}$, O.Rohne $^{32}$, A.Romero $^{45}$, P.Ronchese ${ }^{35}$, E.I.Rosenberg ${ }^{1}$, P.Rosinsky ${ }^{7}$, P.Roudeau ${ }^{19}$, T.Rovelli ${ }^{5}$, Ch.Royon ${ }^{39}$, V.Ruhlmann-Kleider ${ }^{39}$, A.Ruiz ${ }^{40}$, H.Saarikko ${ }^{15}$, Y.Sacquin ${ }^{39}$, A.Sadovsky ${ }^{16}$, G.Sajot ${ }^{14}$, J.Salt ${ }^{49}$, D.Sampsonidis ${ }^{11}$, M.Sannino ${ }^{13}$, H.Schneider $^{17}$, Ph.Schwemling ${ }^{23}$, U.Schwickerath ${ }^{17}$, M.A.E.Schyns ${ }^{52}$, F.Scuri ${ }^{46}$, P.Seager ${ }^{20}$, Y.Sedykh $^{16}$, A.M.Segar ${ }^{34}$, R.Sekulin $^{36}$, R.C.Shellard ${ }^{6}$, A.Sheridan ${ }^{22}$, M.Siebel ${ }^{52}$, L.Simard ${ }^{39}$, F.Simonetto ${ }^{35}$, A.N.Sisakian ${ }^{16}$, T.B.Skaali ${ }^{32}$, G.Smadja ${ }^{25}$, N.Smirnov ${ }^{42}$, O.Smirnova ${ }^{24}$, G.R.Smith ${ }^{36}$, A.Sopczak ${ }^{17}$, R.Sosnowski ${ }^{51}$, T.Spassov ${ }^{21}$, E.Spiriti ${ }^{38}$, P.Sponholz ${ }^{52}$, S.Squarcia ${ }^{13}$, D.Stampfer ${ }^{50}$, C.Stanescu ${ }^{38}$, S.Stanic ${ }^{43}$, S.Stapnes ${ }^{32}$, K.Stevenson ${ }^{34}$, A.Stocchi ${ }^{19}$, J.Strauss ${ }^{50}$, R.Strub ${ }^{10}$, B.Stugu ${ }^{4}$, M.Szczekowski ${ }^{51}$, M.Szeptycka ${ }^{51}$, T.Tabarelli2 ${ }^{27}$, F.Tegenfeldt ${ }^{48}$, F.Terranova ${ }^{27}$, J.Thomas ${ }^{34}$, A.Tilquin ${ }^{26}$, J.Timmermans ${ }^{30}$, N.Tinti ${ }^{5}$, L.G.Tkatchev ${ }^{16}$, S.Todorova ${ }^{10}$, D.Z.Toet ${ }^{30}$, A.Tomaradze ${ }^{2}$, B.Tome ${ }^{21}$, A.Tonazzo ${ }^{27}$, 
L.Tortora ${ }^{38}$, G.Transtromer ${ }^{24}$, D.Treille ${ }^{9}$, G.Tristram ${ }^{8}$, M.Trochimczuk ${ }^{51}$, C.Troncon ${ }^{27}$, A.Tsirou ${ }^{9}$, M-L.Turluer ${ }^{39}$, I.A.Tyapkin ${ }^{16}, \quad$ S.Tzamarias ${ }^{11}, \quad$ B.Ueberschaer ${ }^{52}, \quad$ O.Ullaland $^{9}, \quad$ V.Uvarov ${ }^{42}, \quad$ G.Valenti ${ }^{5}, \quad$ E.Vallazza $^{46}$, G.W.Van Apeldoorn ${ }^{30}$, P.Van Dam ${ }^{30}$, W.K.Van Doninck ${ }^{2}, \quad$ J.Van Eldik ${ }^{30}, \quad$ A.Van Lysebetten ${ }^{2}, \quad$ I.Van Vulpen $^{30}$, N.Vassilopoulos ${ }^{34}$, G.Vegni ${ }^{27}$, L.Ventura ${ }^{35}$, W.Venus ${ }^{36}$, F.Verbeure ${ }^{2}$, M.Verlato ${ }^{35}$, L.S.Vertogradov ${ }^{16}$, V.Verzi ${ }^{37}$,

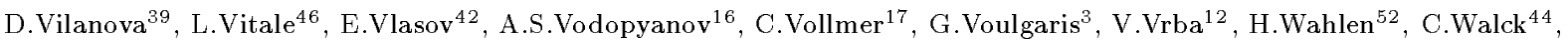
C.Weiser $^{17}$, D.Wicke ${ }^{52}$, J.H.Wickens ${ }^{2}$, G.R.Wilkinson ${ }^{9}$, M.Winter ${ }^{10}$, M.Witek ${ }^{18}$, G.Wolf ${ }^{9}$, J.Yi ${ }^{1}$, O.Yushchenko ${ }^{42}$, A.Zaitsev $^{42}$, A.Zalewska ${ }^{18}$, P.Zalewski $^{51}$, D.Zavrtanik ${ }^{43}$, E.Zevgolatakos ${ }^{11}$, N.I.Zimin ${ }^{16,24}$, G.C.Zucchelli ${ }^{44}$, G.Zumerle $^{35}$

\footnotetext{
${ }^{1}$ Department of Physics and Astronomy, Iowa State University, Ames IA 50011-3160, USA
}

${ }^{2}$ Physics Department, Univ. Instelling Antwerpen, Universiteitsplein 1, BE-2610 Wilrijk, Belgium and IIHE, ULB-VUB, Pleinlaan 2, BE-1050 Brussels, Belgium

and Faculté des Sciences, Univ. de l'Etat Mons, Av. Maistriau 19, BE-7000 Mons, Belgium

${ }^{3}$ Physics Laboratory, University of Athens, Solonos Str. 104, GR-10680 Athens, Greece

${ }^{4}$ Department of Physics, University of Bergen, Allégaten 55, NO-5007 Bergen, Norway

${ }^{5}$ Dipartimento di Fisica, Università di Bologna and INFN, Via Irnerio 46, IT-40126 Bologna, Italy

${ }^{6}$ Centro Brasileiro de Pesquisas Físicas, rua Xavier Sigaud 150, BR-22290 Rio de Janeiro, Brazil and Depto. de Física, Pont. Univ. Católica, C.P. 38071 BR-22453 Rio de Janeiro, Brazil

and Inst. de Física, Univ. Estadual do Rio de Janeiro, rua São Francisco Xavier 524, Rio de Janeiro, Brazil

${ }^{7}$ Comenius University, Faculty of Mathematics and Physics, Mlynska Dolina, SK-84215 Bratislava, Slovakia

${ }^{8}$ Collège de France, Lab. de Physique Corpusculaire, IN2P3-CNRS, FR-75231 Paris Cedex 05, France

${ }^{9}$ CERN, CH-1211 Geneva 23, Switzerland

${ }^{10}$ Institut de Recherches Subatomiques, IN2P3 - CNRS/ULP - BP20, FR-67037 Strasbourg Cedex, France

${ }^{11}$ Institute of Nuclear Physics, N.C.S.R. Demokritos, P.O. Box 60228, GR-15310 Athens, Greece

${ }^{12}$ FZU, Inst. of Phys. of the C.A.S. High Energy Physics Division, Na Slovance 2, CZ-180 40, Praha 8, Czech Republic

${ }^{13}$ Dipartimento di Fisica, Università di Genova and INFN, Via Dodecaneso 33, IT-16146 Genova, Italy

${ }^{14}$ Institut des Sciences Nucléaires, IN2P3-CNRS, Université de Grenoble 1, FR-38026 Grenoble Cedex, France

${ }^{15}$ Helsinki Institute of Physics, HIP, P.O. Box 9, FI-00014 Helsinki, Finland

${ }^{16}$ Joint Institute for Nuclear Research, Dubna, Head Post Office, P.O. Box 79, RU-101 000 Moscow, Russian Federation

${ }^{17}$ Institut für Experimentelle Kernphysik, Universität Karlsruhe, Postfach 6980, DE-76128 Karlsruhe, Germany

${ }^{18}$ Institute of Nuclear Physics and University of Mining and Metalurgy, Ul. Kawiory 26a, PL-30055 Krakow, Poland

${ }^{19}$ Université de Paris-Sud, Lab. de l'Accélérateur Linéaire, IN2P3-CNRS, Bât. 200, FR-91405 Orsay Cedex, France

${ }^{20}$ School of Physics and Chemistry, University of Lancaster, Lancaster LA1 4YB, UK

${ }^{21}$ LIP, IST, FCUL - Av. Elias Garcia, 14-1 ${ }^{\circ}$, PT-1000 Lisboa Codex, Portugal

${ }^{22}$ Department of Physics, University of Liverpool, P.O. Box 147, Liverpool L69 3BX, UK

${ }^{23}$ LPNHE, IN2P3-CNRS, Univ. Paris VI et VII, Tour 33 (RdC), 4 place Jussieu, FR-75252 Paris Cedex 05, France

${ }^{24}$ Department of Physics, University of Lund, Sölvegatan 14, SE-223 63 Lund, Sweden

${ }^{25}$ Université Claude Bernard de Lyon, IPNL, IN2P3-CNRS, FR-69622 Villeurbanne Cedex, France

${ }^{26}$ Univ. d'Aix - Marseille II - CPP, IN2P3-CNRS, FR-13288 Marseille Cedex 09, France

${ }^{27}$ Dipartimento di Fisica, Università di Milano and INFN, Via Celoria 16, IT-20133 Milan, Italy

${ }^{28}$ Niels Bohr Institute, Blegdamsvej 17, DK-2100 Copenhagen Ø, Denmark

${ }^{29} \mathrm{NC}$, Nuclear Centre of MFF, Charles University, Areal MFF, V Holesovickach 2, CZ-180 00, Praha 8, Czech Republic

${ }^{30}$ NIKHEF, Postbus 41882, NL-1009 DB Amsterdam, The Netherlands

${ }^{31}$ National Technical University, Physics Department, Zografou Campus, GR-15773 Athens, Greece

32 Physics Department, University of Oslo, Blindern, NO-1000 Oslo 3, Norway

${ }^{33}$ Dpto. Fisica, Univ. Oviedo, Avda. Calvo Sotelo s/n, ES-33007 Oviedo, Spain

${ }^{34}$ Department of Physics, University of Oxford, Keble Road, Oxford OX1 3RH, UK

${ }^{35}$ Dipartimento di Fisica, Università di Padova and INFN, Via Marzolo 8, IT-35131 Padua, Italy

${ }^{36}$ Rutherford Appleton Laboratory, Chilton, Didcot OX11 OQX, UK

${ }^{37}$ Dipartimento di Fisica, Università di Roma II and INFN, Tor Vergata, IT-00173 Rome, Italy

${ }^{38}$ Dipartimento di Fisica, Università di Roma III and INFN, Via della Vasca Navale 84, IT-00146 Rome, Italy

${ }^{39}$ DAPNIA/Service de Physique des Particules, CEA-Saclay, FR-91191 Gif-sur-Yvette Cedex, France

${ }^{40}$ Instituto de Fisica de Cantabria (CSIC-UC), Avda. los Castros s/n, ES-39006 Santander, Spain

${ }^{41}$ Dipartimento di Fisica, Università degli Studi di Roma La Sapienza, Piazzale Aldo Moro 2, IT-00185 Rome, Italy

${ }^{42}$ Inst. for High Energy Physics, Serpukov P.O. Box 35, Protvino, (Moscow Region), Russian Federation

43 J. Stefan Institute, Jamova 39, SI-1000 Ljubljana, Slovenia and Department of Astroparticle Physics, School of Environmental Sciences, Kostanjeviska 16a, Nova Gorica, SI-5000 Slovenia, and Department of Physics, University of Ljubljana, SI-1000 Ljubljana, Slovenia

${ }^{44}$ Fysikum, Stockholm University, Box 6730, SE-113 85 Stockholm, Sweden

${ }^{45}$ Dipartimento di Fisica Sperimentale, Università di Torino and INFN, Via P. Giuria 1, IT-10125 Turin, Italy

${ }^{46}$ Dipartimento di Fisica, Università di Trieste and INFN, Via A. Valerio 2, IT-34127 Trieste, Italy and Istituto di Fisica, Università di Udine, IT-33100 Udine, Italy

${ }^{47}$ Univ. Federal do Rio de Janeiro, C.P. 68528 Cidade Univ., Ilha do Fundão BR-21945-970 Rio de Janeiro, Brazil

${ }^{48}$ Department of Radiation Sciences, University of Uppsala, P.O. Box 535, SE-751 21 Uppsala, Sweden

${ }^{49}$ IFIC, Valencia-CSIC, and D.F.A.M.N., U. de Valencia, Avda. Dr. Moliner 50, ES-46100 Burjassot (Valencia), Spain

${ }^{50}$ Institut für Hochenergiephysik, Österr. Akad. d. Wissensch., Nikolsdorfergasse 18, AT-1050 Vienna, Austria

${ }^{51}$ Inst. Nuclear Studies and University of Warsaw, Ul. Hoza 69, PL-00681 Warsaw, Poland

${ }^{52}$ Fachbereich Physik, University of Wuppertal, Postfach 100 127, DE-42097 Wuppertal, Germany

${ }^{53}$ On leave of absence from IHEP Serpukhov

54 Now at University of Florida 


\section{Introduction}

The relative decay width of the $\mathrm{Z}$ into b-quarks, $R_{\mathrm{b}}^{0}=\Gamma_{\mathrm{b} \overline{\mathrm{b}}} / \Gamma_{\text {had }}$, plays an important role amongst the observables measured with high precision at LEP and SLC. The other observables are mainly sensitive to electroweak radiative corrections in the Z-propagator, setting important constraints on, for example, the Higgs boson mass [1]. However, these corrections mainly cancel in the ratio of two partial widths and only those to the Zq $\bar{q}$ vertex remain, which are naturally enhanced with the fermion mass. Since the b-quark is the isospin partner of the very heavy top-quark, the $\mathrm{Zb} \overline{\mathrm{b}}$ vertex is especially interesting. As an example, within supersymmetry and for a certain range of the model's parameters, effects due to the existence of stop-quarks or charginos could lead to observable changes of $R_{\mathrm{b}}^{0}$ with respect to the Standard Model [2].

The presently published results from the LEP collaborations and SLD reach an overall accuracy of $0.5 \%[3-9]$; this accuracy is marginal for observing possible predicted deviations from the Standard Model. This paper updates and supersedes the previous DELPHI result and exploits the full statistics and understanding of the detector behaviour, improving significantly the precision of the previous measurement.

Experimentally, $R_{\mathrm{b}}^{0}$ can be obtained with only very small corrections from the ratio of cross-sections $R_{\mathrm{b}}=\sigma\left(\mathrm{e}^{+} \mathrm{e}^{-} \rightarrow \mathrm{b} \overline{\mathrm{b}}\right) / \sigma\left(\mathrm{e}^{+} \mathrm{e}^{-} \rightarrow\right.$ hadrons $)$. This paper presents measurements of $R_{\mathrm{b}}$ using about 3.4 million hadronic events taken in the years 1992 to 1995 with the DELPHI detector at LEP. The data in 1992 and 1994 were collected at the centre of the $\mathrm{Z}$ peak; in 1993 and 1995, scans across the $\mathrm{Z}$ peak were performed.

All analyses compare the rates of events where only one of the b-quarks has been identified to those where both b's have been tagged, from which $R_{\mathrm{b}}$ can be measured together with the b-tagging efficiency. Systematic uncertainties due to the charm background and to hemisphere correlations have been considerably reduced with respect to previous analyses [5] due to improved tracking algorithms in the charged track reconstruction, to the use of new variables for the identification of b-quarks and to a new method for reconstructing the primary vertex. One analysis (the multivariate analysis) uses, in addition to the highly efficient and pure b-tag, additional tags for b-, c- and light quarks; all efficiencies apart from the background efficiencies of the primary b-tag are measured from data, so that the new tags reduce the statistical error without increasing the systematic uncertainties.

This paper is organised as follows. After a description of the relevant characteristics of the detector and of the track and event selection, sections 4, 5 and 6 describe the different analyses: the enhanced impact parameter analysis, the multivariate analysis and the secondary vertex neural network analysis. Section 7 describes the use of the enhanced impact parameter tag to study the energy dependence of $R_{\mathrm{b}}$ with the data collected during the scan across the $\mathrm{Z}$ peak in 1993 and 1995. Section 8 compares the result of this paper with the previous published DELPHI result and the last section contains a summary.

\section{The DELPHI Detector}

The DELPHI detector and its performance have been described in detail elsewhere $[10,11]$. Only the details most relevant to this analysis are mentioned here, including the upgraded Microvertex Detector, installed in spring 1994, that allowed high values of purity and efficiency in the identification of the b-quarks to be reached. 
In the barrel region, the charged particle tracks are measured by a set of cylindrical tracking detectors whose axes are parallel to the $1.2 \mathrm{~T}$ solenoidal magnetic field and to the beam direction.

The innermost one is the Microvertex Detector (VD), which is located between the LEP beam pipe and the Inner Detector (ID) $[12,13]$. The DELPHI Microvertex Detector used from 1991 to 1993 [12] was composed of 3 layers of single-sided silicon microstrip detectors at radii of $6.3,9$ and $11 \mathrm{~cm}$ from the beam line, respectively called the closer, inner and outer layers. To improve the performance of the detector in tracking and especially in the identification of b-hadrons, in 1994 it was upgraded using double-sided silicon detectors allowing three-dimensional impact parameter reconstruction. The microstrip detectors of the closer and outer layers provide hits in both the $R \phi$ and the $R z$ planes ${ }^{1}$, while for the inner layer only the $R \phi$ coordinate is measured. For polar angles of $44^{\circ} \leq \theta \leq 136^{\circ}$ a track crosses all three silicon layers of the VD. The closer layer covers the polar region between $25^{\circ}$ and $155^{\circ}$.

The measured intrinsic resolution is about $8 \mu \mathrm{m}$ for the $R \phi$ coordinate for both the old and the upgraded VD, while for $R z$ it depends on the incident polar angle of the track and reaches about $9 \mu \mathrm{m}$ for tracks perpendicular to the modules. For tracks with hits in all three $R \phi$ VD layers, the impact parameter resolution is $\sigma_{R \phi}^{2}=\left(\left(61 /\left(P \sin ^{3 / 2} \theta\right)\right)^{2}+\right.$ $\left.20^{2}\right) \mu \mathrm{m}^{2}$ for both the old and the upgraded VD; for tracks with hits in both $R z$ layers and with $\theta \approx 90^{\circ}, \sigma_{R z}^{2}=\left(\left(67 /\left(P \sin ^{5 / 2} \theta\right)\right)^{2}+33^{2}\right) \mu \mathrm{m}^{2}$.

The Time Projection Chamber (TPC) is the main tracking device and is a cylinder of length $3 \mathrm{~m}$, inner radius $30 \mathrm{~cm}$ and outer radius $122 \mathrm{~cm}$. Between polar angles of $39^{\circ}$ and $141^{\circ}$, tracks are reconstructed using up to 16 space points. Outside this region $\left(21^{\circ}\right.$ to $39^{\circ}$ and $141^{\circ}$ to $159^{\circ}$ ), tracks can be reconstructed using at least 4 space points.

Additional precise $R \phi$ measurements are provided at larger and smaller radii by the Outer and Inner detectors respectively. The Outer Detector (OD) has five layers of drift cells at radii between 198 and $206 \mathrm{~cm}$ and covers polar angles from $42^{\circ}$ to $138^{\circ}$. The Inner Detector (ID) is a cylindrical drift chamber having inner radius of $12 \mathrm{~cm}$ and outer radius of $28 \mathrm{~cm}$ and covers polar angles between $29^{\circ}$ and $151^{\circ}$. It contains a jet chamber section providing $24 R \phi$ coordinates, surrounded by five layers of proportional chambers giving both $R \phi$ and $z$ coordinates.

The barrel electromagnetic calorimeter (HPC) covers polar angles between $42^{\circ}$ and $138^{\circ}$. It is a gas-sampling device which provides complete three-dimensional charge information in the same way as a time projection chamber. The excellent granularity allows good separation between close particles in three dimensions and hence good electron identification even inside jets.

In the forward region the tracking is complemented by two sets of planar drift chambers (FCA and FCB), at distances of $z= \pm 165 \mathrm{~cm}$ and $z= \pm 275 \mathrm{~cm}$ from the interaction point. A lead glass calorimeter (EMF) is used to reconstruct electromagnetic energy in the forward region.

Muon identification in the barrel region is based on a set of muon chambers (MUB), covering polar angles between $53^{\circ}$ and $127^{\circ}$. In the forward region the muon identification is provided using two sets of planar drift chambers (MUF) covering the angular region between $11^{\circ}$ and $45^{\circ}$.

\footnotetext{
${ }^{1}$ In the DELPHI coordinate system, $z$ is along the beam line, $\phi$ and $R$ are the azimuthal angle and radius in the $x y$ plane, and $\theta$ is the polar angle with respect to the $z$ axis.
} 


\section{Event Selection}

The criteria to select charged particles and to identify hadronic Z decays were similar to those described in [5]. Charged particles were accepted if:

- their polar angle was between $20^{\circ}$ and $160^{\circ}$,

- their track length was larger than $30 \mathrm{~cm}$,

- their impact parameter relative to the interaction point was less than $5 \mathrm{~cm}$ in the plane perpendicular to the beam direction and less than $8 \mathrm{~cm}$ along the beam direction,

- their momentum was larger than $200 \mathrm{MeV} / c$ with relative error less than $100 \%$.

Neutral particles detected in the HPC were required to have measured energy larger than $700 \mathrm{MeV}$ and those detected in the EMF greater than $400 \mathrm{MeV}$.

Events were then selected by requiring:

- at least 6 reconstructed charged particles,

- the summed energy of the charged particles had to be larger than $15 \%$ of the centreof-mass energy, with at least $3 \%$ of it in each of the forward and backward hemispheres with respect to the beam axis.

The efficiency to find hadronic $\mathrm{Z}$ decays with these cuts was about $95 \%$ and all backgrounds were below $0.1 \%$.

About 1.3 million hadronic $\mathrm{Z}$ decays were selected with the two-dimensional VD in 1992 and 1993, and 2.1 million hadronic Z decays from the 1994 and 1995 data samples with the three-dimensional VD. The ratio of the cross-section $Z \rightarrow b \bar{b}$ to the total hadronic cross-section varies very little at centre-of-mass energies close to the $\mathrm{Z}$ mass. Thus no selection on the centre-of-mass energy was made in 1993 and 1995 . However the validity of this assumption has been tested (see section 7).

As the VD is essential for efficient b-tagging, all methods were limited to events that have most of the tracks inside its acceptance. For this reason a cut of $\left|\cos \theta_{\text {thrust }}\right|<0.65$ was applied, keeping about $60 \%$ of the events. The bias towards b events in the selected sample was found to be small, $(1.51 \pm 0.09) \cdot 10^{-3}$, and was corrected for; its uncertainty is dominated by Monte Carlo statistics.

A sample about twice the data statistics of $Z \rightarrow q \bar{q}$ events was generated using the Lund parton shower Monte Carlo JETSET 7.3 [14] (with parameters optimized by DELPHI [15]) and the detector response simulated using DELSIM [11]. In addition, dedicated samples of $Z \rightarrow b \bar{b}$ events were generated. The simulated events were passed through the same analysis chain as the real ones.

\section{The Enhanced Impact Parameter Analysis}

\subsection{The method}

Events are divided into hemispheres using the plane perpendicular to the thrust axis. If $f_{H}$ is the fraction of hemispheres tagged as $\mathrm{b}$ and $f_{E}$ is the fraction of events in which both hemispheres are tagged, $R_{\mathrm{b}}$ can be extracted from $f_{H}$ and $f_{E}$ :

$$
\begin{aligned}
& f_{H}=R_{\mathrm{b}} \cdot \epsilon_{\mathrm{b}}+R_{\mathrm{c}} \cdot \epsilon_{\mathrm{c}}+\left(1-R_{\mathrm{b}}-R_{\mathrm{c}}\right) \cdot \epsilon_{\mathrm{uds}} \\
& f_{E}=R_{\mathrm{b}} \cdot \epsilon_{\mathrm{b}}^{2} \cdot(1+\rho)+R_{\mathrm{c}} \cdot \epsilon_{\mathrm{c}}^{2}+\left(1-R_{\mathrm{b}}-R_{\mathrm{c}}\right) \cdot \epsilon_{\mathrm{uds}}^{2},
\end{aligned}
$$


where $\epsilon_{\mathrm{q}}$ is the efficiency to tag a hemisphere originating from a primary quark q (=uds, $c, b)$ and the coefficient $\rho$ accounts for hemisphere correlations in the tagging efficiencies for b-quarks. For the other quark species these correlation factors can safely be neglected due to the very high b-purity reached. If $\rho, \epsilon_{\mathrm{c}}$ and $\epsilon_{\mathrm{uds}}$ are calculated from the simulation and $R_{\mathrm{c}}$ is imposed from other measurements or from the Standard Model, $R_{\mathrm{b}}$ and $\epsilon_{\mathrm{b}}$ can be measured simultaneously from the data. Precise knowledge of the details of b-hadron decays is thus not required.

Compared with the previous DELPHI publication [5], a new b-tagging variable is used (see section 4.2). The main ingredient is the measurement of the track impact parameters, but now complemented by additional information such as the invariant mass and the energy of particles fitted to a secondary vertex. Where to cut in this variable is arbitrary and the cut chosen for the results was that which minimised the total error. This resulted in very high b-purity. Results are given as a function of the b-purity and b-efficiency of the data sample.

Two contributions to the systematic error come from the background mistagging probabilities $\epsilon_{\mathrm{c}}$ and $\epsilon_{\mathrm{uds}}$ (i.e. the fraction of light quark hemispheres that are tagged as $\mathrm{b}$ - see section 4.3), and the hemisphere correlation coefficient $\rho$. The former were substantially reduced using the purer tag. From the previous analysis [5], it was estimated that the major contribution to the second effect came from the common primary vertex for the two hemispheres of the event. The correlation was substantially reduced by computing a separate primary vertex for each hemisphere. The remaining correlation is discussed in section 4.5 .

For the extraction of $R_{\mathrm{b}}$ with such a method, a good description of the data by the simulation for the udsc-quarks is required. For this reason a fine tuning of the $R \phi$ and $R z$ impact parameter distributions in the simulation was developed and applied [16]. This led to substantially smaller uncertainties due to the understanding of the detector resolution.

\subsection{Reconstruction of secondary vertices}

The primary vertex for the hemisphere was reconstructed using the tracks in that hemisphere with at least one measurement in the VD, and the constraint of the beamspot ${ }^{2}$. The main features of the fitting procedure are described in detail in [5].

A search for secondary vertices is made within each jet. In the first step all possible combinations of pairs of tracks are selected as secondary vertex candidates if they result in a common vertex with the $\chi^{2}$ of the fit less than 4 . A track from the same jet is added to the given vertex candidate if the change of $\chi^{2}$ of the vertex fit with this addition does not exceed 5 . The track with the smallest change of $\chi^{2}$ is added first. The vertex candidate is rejected if the distance to the primary vertex divided by its error does not exceed 4. For the data sample with the double-sided VD, at least 2 tracks are required to have measurements in the $R \phi$ and $R z$ planes of the VD. For the data sample with the single-sided VD, the fit is performed in the $R \phi$ plane only and at least 3 tracks are required for the vertex candidate.

The direction of the vertex candidate is defined as the vector from the primary to the secondary vertex. Any track from the same jet is added to the vertex candidate if its distance to the direction of the vertex in space divided by its error is less than 3 . With such addition, tracks from b-hadrons decays with 2 separate vertices are recuperated. Even though the point of the decay of the c-hadron can be far from that of the parent

\footnotetext{
${ }^{2}$ Defined by the interaction points of a few hundred events within the same running period. Typical dimensions of the beam spot are: $120 \mu \mathrm{m}$ horizontally, $10 \mu \mathrm{m}$ vertically and $2 \mathrm{~cm}$ along the beam.
} 
b-hadron, its direction follows that of the b-hadron so that the tracks of its decay have a small distance in space to the direction of the b-hadron. This procedure is not used for the data sample with the single-sided VD where only $R \phi$ measurements are available.

Any two vertex candidates are combined as a single one if the angle between their directions is less than $0.20 \mathrm{rad}$. By this procedure, the two separate vertices of the cascade $(\mathrm{B} \rightarrow \mathrm{D})$ decay are combined. After this, the final fit of the vertex is performed using the selected group of tracks. The tracks are kept in the final vertex candidate either if they are included in the vertex fit or if they have a small distance to the vertex candidate direction in the space.

To estimate the flight direction of the b-hadron, the sum of the momenta of all the tracks included in the vertex candidate is computed. Also included in this sum are the momenta of other charged or neutral particles with rapidity with respect to the vertex direction larger than 2 . Using the resulting estimate of the total momentum of the b-hadron and the vertex positions, the impact parameter of the vertex candidate is computed with respect to the primary vertex (in space for the double-sided VD and in the $R \phi$ plane for the single-sided VD). The impact parameter of the vertex candidate divided by its error is required to be less than 4.4 .

The positive lifetime probability $[17,5]$ (see section 4.3) is computed using all charged tracks included in the vertex candidate, and the vertex candidate is rejected if this probability exceeds 0.01 .

The remaining candidates are defined as the reconstructed secondary vertices.

\subsection{Tagging technique}

Events with weakly decaying b-hadrons are significantly different from those containing only lighter quarks. b-hadrons have a large mass, a long lifetime and a high decay multiplicity, they take more energy from the initial quark than do light hadrons, etc. However in previous DELPHI measurements of $R_{\mathrm{b}}$, only their long lifetime and the high decay multiplicity were used for the tagging [5]. In this paper we describe a method of b-tagging which combines into a single variable all these differences of the b-hadrons with respect to other particles. The application of this method gives a significant improvement of the b-tagging efficiency with respect to the lifetime tag used previously.

First, the particles are clustered into jets (using the JADE algorithm with $y_{\min }=0.01$ ), and a tagging variable is calculated for each. For a hemisphere containing just one jet, the tagging variable is simply that for the single jet. If a hemisphere contains more than one jet, the jet with the highest probability of coming from a b-quark is used.

In this method all discriminating variables are defined for jets with reconstructed secondary vertices (see section 4.2 ); jets without a reconstructed secondary vertex are not considered further. Such a condition allows properties specific to b-hadrons to be used for the tagging and allows the separation of their decay products from those particles coming from b-quark hadronization. In addition, the requirement that jets have reconstructed secondary vertices is by itself a good b-quark selection as it removes a significant part of the background. Thus hemispheres that would be tagged due to badly measured tracks with large impact parameters can be rejected by the vertex requirement. Because of the requirement of a reconstructed secondary vertex, the purity of b-hadrons in jets is about $85 \%$ with a selection efficiency of almost $50 \%$, before any further requirement is imposed.

The description of the discriminating variables is as follows.

The jet lifetime probability, $P_{j}^{+}$, is constructed from the positively signed impact parameters of the tracks included in a jet and corresponds to the probability that a given 
group of tracks are compatible with the primary vertex $[17,5]$. For jets with b-hadrons, this probability is usually very small due to the significant impact parameters of tracks from long-lived $\mathrm{B}$ decays. However, jets with c-quarks can also have low values of $P_{j}^{+}$ because of the non-zero lifetime of $\mathrm{D}$-mesons, which limits the performance of the lifetime tag. The distribution of $-\log _{10}\left(P_{j}^{+}\right)$for different quark flavours is shown in figure 1 a.

The distribution of the effective mass of particles included in the secondary vertex, $M_{s}$, is shown in figure $1 \mathrm{~b}$. The mass of the secondary vertex for c-jets is limited by the mass of $\mathrm{D}$-mesons, and above $M_{s}=1.8 \mathrm{GeV} / c^{2}$ the number of c-jets decreases sharply, while for b-jets the mass distribution extends up to $5 \mathrm{GeV} / c^{2}$. The mass of the pion is assumed for all particles.

The distribution of the rapidity of particles included in the secondary vertex with respect to the jet direction, $R_{s}^{t r}$, is shown in figure 1c. Although a b-hadron has on average higher energy than a $\mathrm{D}$-meson from a c-jet, the rapidities of particles from a B decay are usually less than those from a c-quark decay. This is explained by the higher mass of the b-hadron and the larger multiplicity of its decays. The secondary vertices in light quark jets are mainly the result of wrongly measured tracks. The wrong measurements occur due to multiple scattering in the detector, interaction in the material, etc so that particles included in the secondary vertices of light quark jets are usually soft and their rapidity distribution is shifted to lower values.

The distribution of the fraction of the energy of a jet carried by charged particles included in the secondary vertex, $X_{s}^{c h}$, for different quark types is shown in figure 1d. In the case of b-hadrons, when almost all particles included in the secondary vertex come from the B decay, the distribution of $X_{s}^{c h}$ is determined by the fragmentation function $f(\mathrm{~b} \rightarrow B)$. The same is valid for c-quark jets where the distribution of $X_{s}^{c h}$ is determined by $f(\mathrm{c} \rightarrow D)$, which is softer than $f(\mathrm{~b} \rightarrow B)$. In light quark jets, the energy at the secondary vertex is much less than that in b-quark jets, as explained above.

For the combination of the discriminating variables, the following quantity is defined:

$$
y=n_{\mathrm{c}} \cdot \prod_{i} \frac{f_{i}^{\mathrm{c}}\left(x_{i}\right)}{f_{i}^{\mathrm{b}}\left(x_{i}\right)}+n_{\mathrm{q}} \cdot \prod_{i} \frac{f_{i}^{\mathrm{q}}\left(x_{i}\right)}{f_{i}^{\mathrm{b}}\left(x_{i}\right)}=n_{\mathrm{c}} \cdot \prod_{i} y_{i}^{\mathrm{c}}+n_{\mathrm{q}} \cdot \prod_{i} y_{i}^{\mathrm{q}},
$$

where $n_{\mathrm{c}}$ and $n_{\mathrm{q}}(\mathrm{q}=\mathrm{uds})$ are the normalised numbers of jets with a reconstructed secondary vertex in $c \bar{c}$ and $q \bar{q}$ events respectively $\left(n_{\mathrm{c}}+n_{\mathrm{q}}=1\right)$ and $f_{i}^{\mathrm{q}}\left(x_{i}\right), f_{i}^{\mathrm{c}}\left(x_{i}\right), f_{i}^{\mathrm{b}}\left(x_{i}\right)$ are probability density functions of the variable $x_{i}$ in uds-, c- and b-quark jets. All of these quantities are taken from simulation.

The products in (2) run over all tagging variables of a given jet. The variable $R_{s}^{t r}$ is defined for each particle included in the secondary vertex and so the corresponding ratio of probabilities for each particle enters in equation (2). For the ratios $y_{i}^{\mathrm{c}}\left(x_{i}\right)=f_{i}^{\mathrm{c}}\left(x_{i}\right) / f_{i}^{\mathrm{b}}\left(x_{i}\right)$ and $y_{i}^{\mathrm{q}}\left(x_{i}\right)=f_{i}^{\mathrm{q}}\left(x_{i}\right) / f_{i}^{\mathrm{b}}\left(x_{i}\right)$ we use smooth functions which are obtained from a fit of the ratios of corresponding distributions. The jet is tagged as containing a b-quark if $y \leq y_{0}$, where the value $y_{0}$ can be varied to select the desired purity or efficiency of tagging.

Figure 2 shows the tagging efficiency versus purity of the selected sample in simulation for different combinations of discriminating variables with the double-sided VD. It can be seen that the addition of each new variable improves the tagging performance.

The enhanced tagging in comparison with the simple lifetime tag $P_{j}^{+}$suppresses the background by more than a factor 3 for a b-tagging efficiency of $30 \%$ and about 6 times for a b-tagging efficiency of $20 \%$. A very pure b sample with purity more than $99.5 \%$ can be obtained with a b-efficiency of $20 \%$.

All distributions for this tagging method are taken from simulation, so that a check of their agreement with data is important for its successful application. For a measurement 


\section{DELPHI}
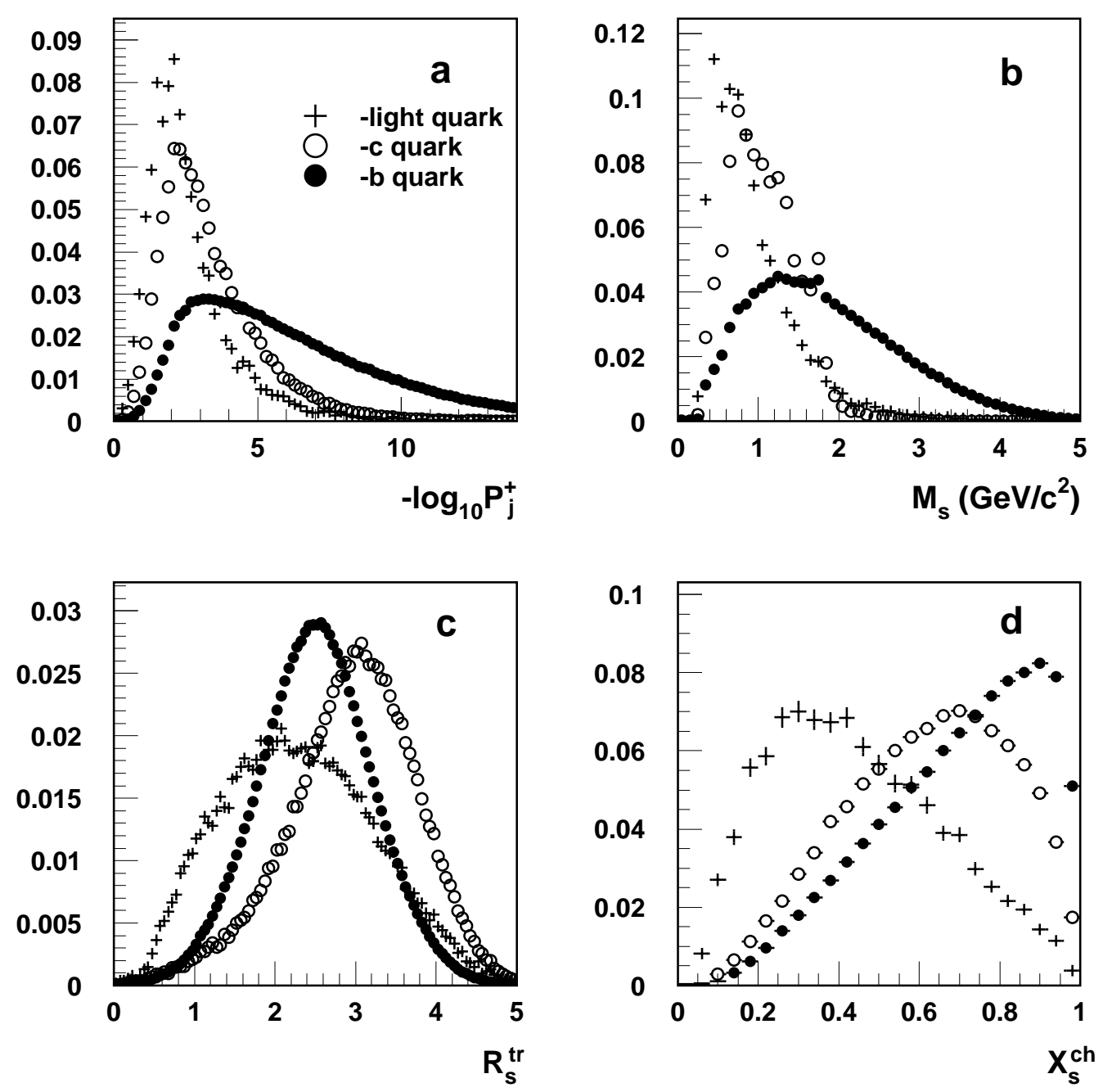

Figure 1: Distributions of discriminating variables from simulation used in the enhanced impact parameter tagging, as determined from simulation. The figures show for the different quark flavours: a) the jet lifetime probability ; b) the effective mass of particles included in the reconstructed secondary vertex in $\mathrm{GeV} / \mathrm{c}^{2}$; c) the rapidity of tracks included in the reconstructed secondary vertex; d) the fraction of the jet energy carried by the charged particles at the reconstructed secondary vertex. 


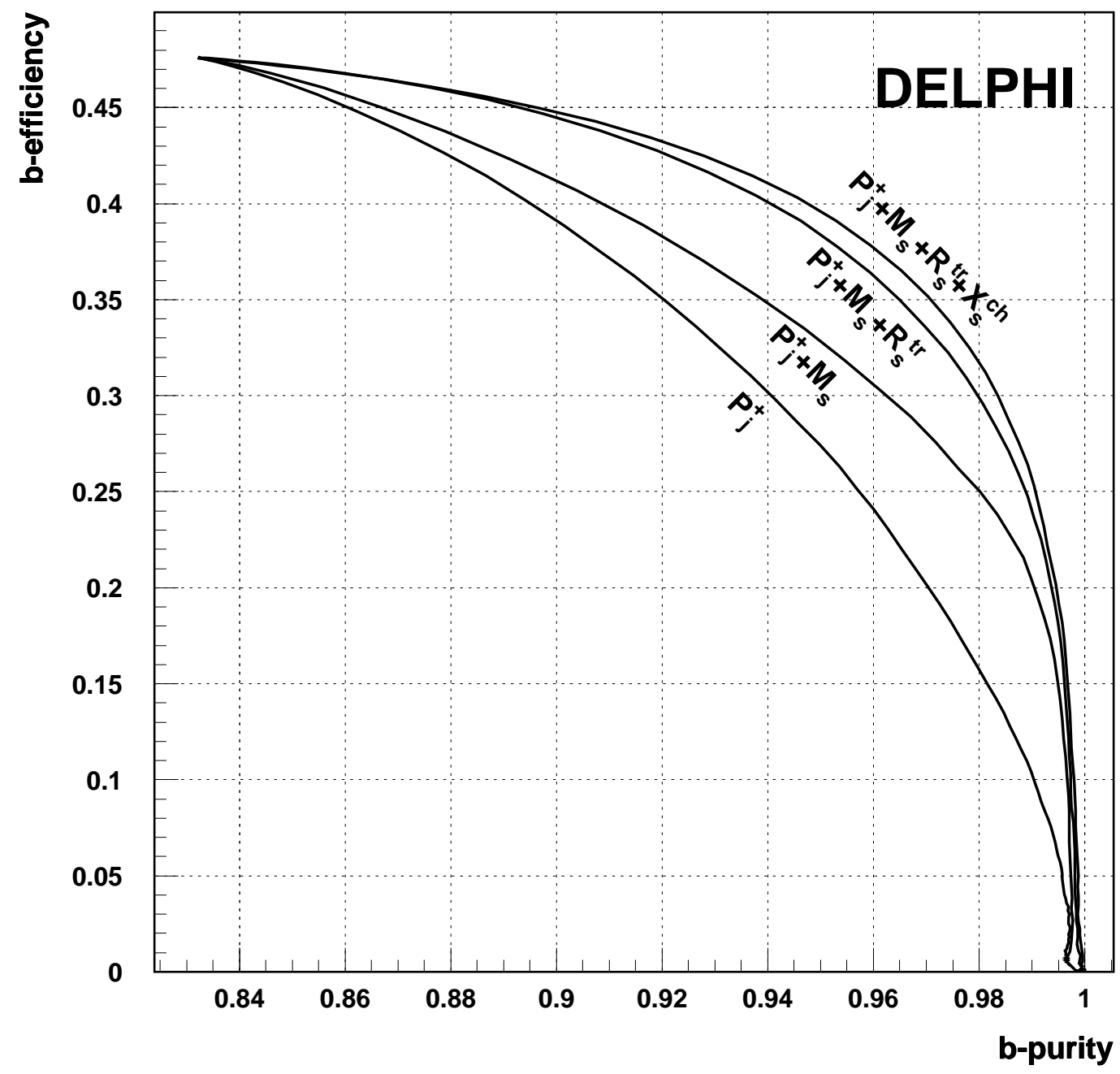

Figure 2: b-tagging hemisphere efficiency versus purity for the enhanced impact parameter method, for different combinations of discriminating variables using the double-sided VD. The presence of a secondary vertex is always required; this already produces a b-purity of $83.5 \%$ without any further selections. 
of $R_{\mathrm{b}}$, only the agreement of background distributions needs to be verified since the efficiency of b-quark tagging is taken from data.

The high purity of the tagged sample allows the extraction from data of the distributions of the discriminating variables for background and the comparison of them with those used in the simulation. b-hadrons in one hemisphere are tagged with a high purity of about $99 \%$ to give a clean and almost uncontaminated sample of b-hadrons in the opposite hemisphere. The distributions of the discriminating variables in such hemispheres can be subtracted after appropriate normalisation from the corresponding distributions in the untagged sample of jets with secondary vertices.

The comparison of these distributions in data and in simulation is shown in figure 3. Good agreement in the background description for all variables used in the tagging can be seen. Finally, figure 4 shows the comparison of distributions of the enhanced tagging variable $-\log _{10} y$, where $y$ is defined by $(2)$.

\subsection{Light and charm quark mistagging probabilities}

The analysis was performed at many different values of the b-tagging efficiency and purity. The minimum total error (i.e. the sum in quadrature of the statistical and systematic errors) in the 1994-1995 data analysis was obtained for $\epsilon_{\mathrm{b}}=32.1 \%$, i.e. for a cut on the variable $-\log _{10} y \geq 1$. For the two-dimensional VD in 1992 and 1993 a lower efficiency of $28 \%$ is obtained for the same purity. The multivariate analysis (see Section 5), which uses the enhanced impact parameter tag as its primary tag, has its optimum error at slightly harder cut values, namely $-\log _{10} y \geq 1.2$ for $1994-1995$ and $-\log _{10} y \geq 0.6$ for $1992-1993$. Since this section is mainly meant to illustrate the relevant features for the multivariate analysis, which provides the main result for this paper, all numbers are presented using these cuts.

At these chosen working points, the mistagging probabilities for uds- and c-quarks were estimated using the simulation to be

$$
\begin{aligned}
\epsilon_{\mathrm{uds}} & =0.00050 \pm 0.00006 \\
\epsilon_{\mathrm{c}} & =0.00381 \pm 0.00025
\end{aligned}
$$

for 1993 and 1992, while they are

$$
\begin{aligned}
\epsilon_{\mathrm{uds}} & =0.00052 \pm 0.00008 \\
\epsilon_{\mathrm{c}} & =0.00376 \pm 0.00027
\end{aligned}
$$

for 1994 and 1995. The breakdown of the errors is given in table 1.

For the values and uncertainties of most physical quantities, the recommendations of the LEPHF group [18] have been followed.

An especially complicated issue is the dependence of $\epsilon_{\mathrm{c}}$ on the charmed hadron decay modes. For the b-tag used in this analysis, about $45 \%$ of the tagged c-hemispheres contain a $\mathrm{D}^{0}$, and similarly for a charged $D$. (This is because even though more $\mathrm{D}^{0}$ than charged $D$ s are produced, a smaller fraction of $\mathrm{D}^{0}$ are tagged because of their shorter lifetime.) About $10 \%$ contain a $\mathrm{D}_{\mathrm{s}}$ and only $1 \%$ a charmed baryon. Details of the charmed baryon decays are therefore not important for the understanding of $\epsilon_{\mathrm{c}}$. Because of the much worse knowledge of $\mathrm{D}_{\mathrm{s}}$ decays compared to $\mathrm{D}^{0}$ and $\mathrm{D}^{+}$, the uncertainties due to the three charmed mesons are of comparable size. Two main features of D-meson decays are relevant for the charm mistagging probability: the charged decay multiplicity and the multiplicity of neutral particles. Mistagging increases with charged multiplicity because 


\section{DELPHI}
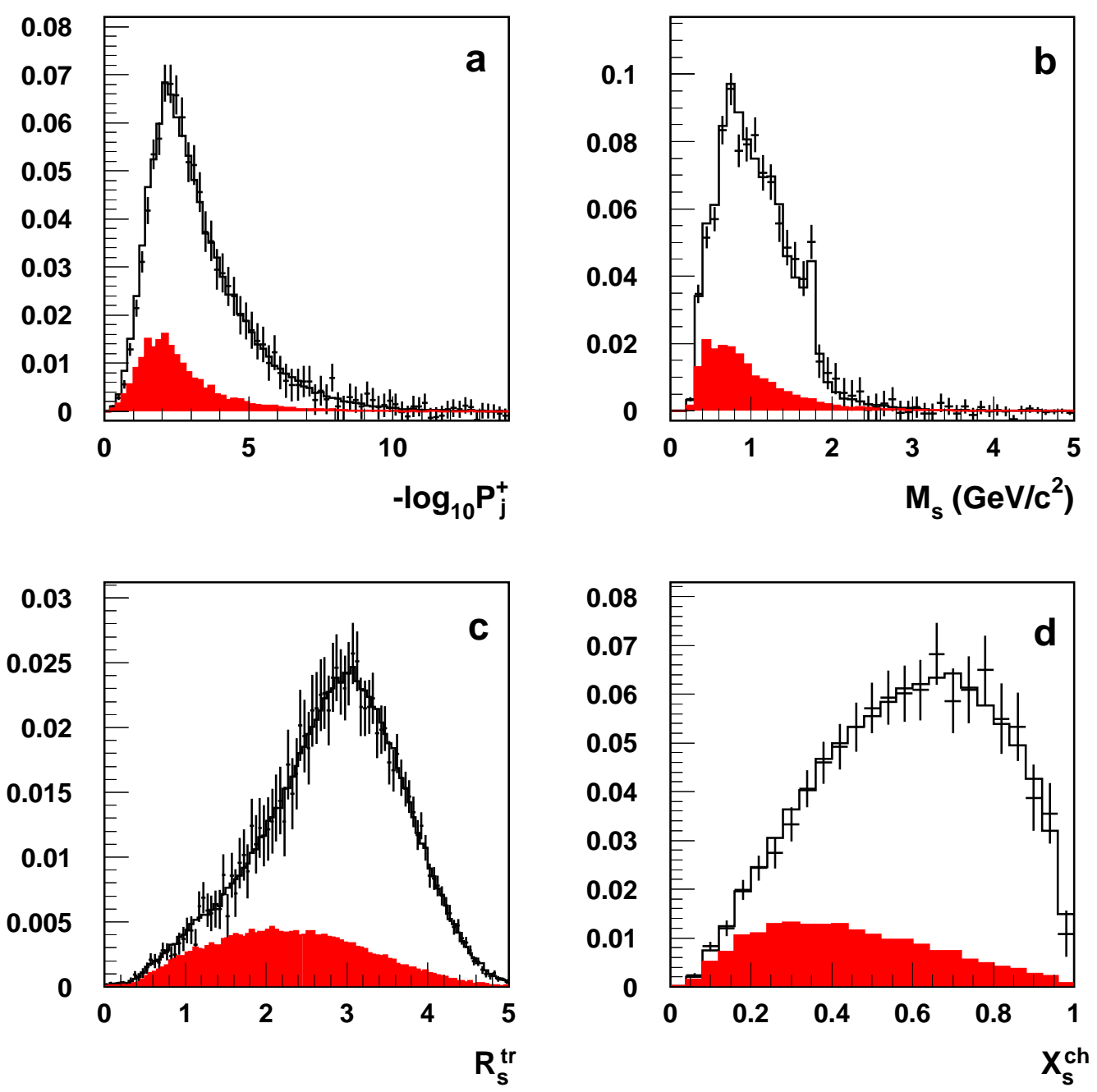

Figure 3: Distribution of discriminating variables for background $(\mathrm{u}, \mathrm{d}, \mathrm{s}, \mathrm{c})$ jets. The points with errors are from the data and the histogram is the simulation prediction. The contribution of uds-quark jets is shown as the filled histograms. The figures show a) the jet lifetime probability; b) the effective mass of particles included in the reconstructed secondary vertex in $\mathrm{GeV} / c^{2}$; c) the rapidity of tracks included in the reconstructed secondary vertex; d) the fraction of the jet energy carried by the charged particles of the reconstructed secondary vertex. 

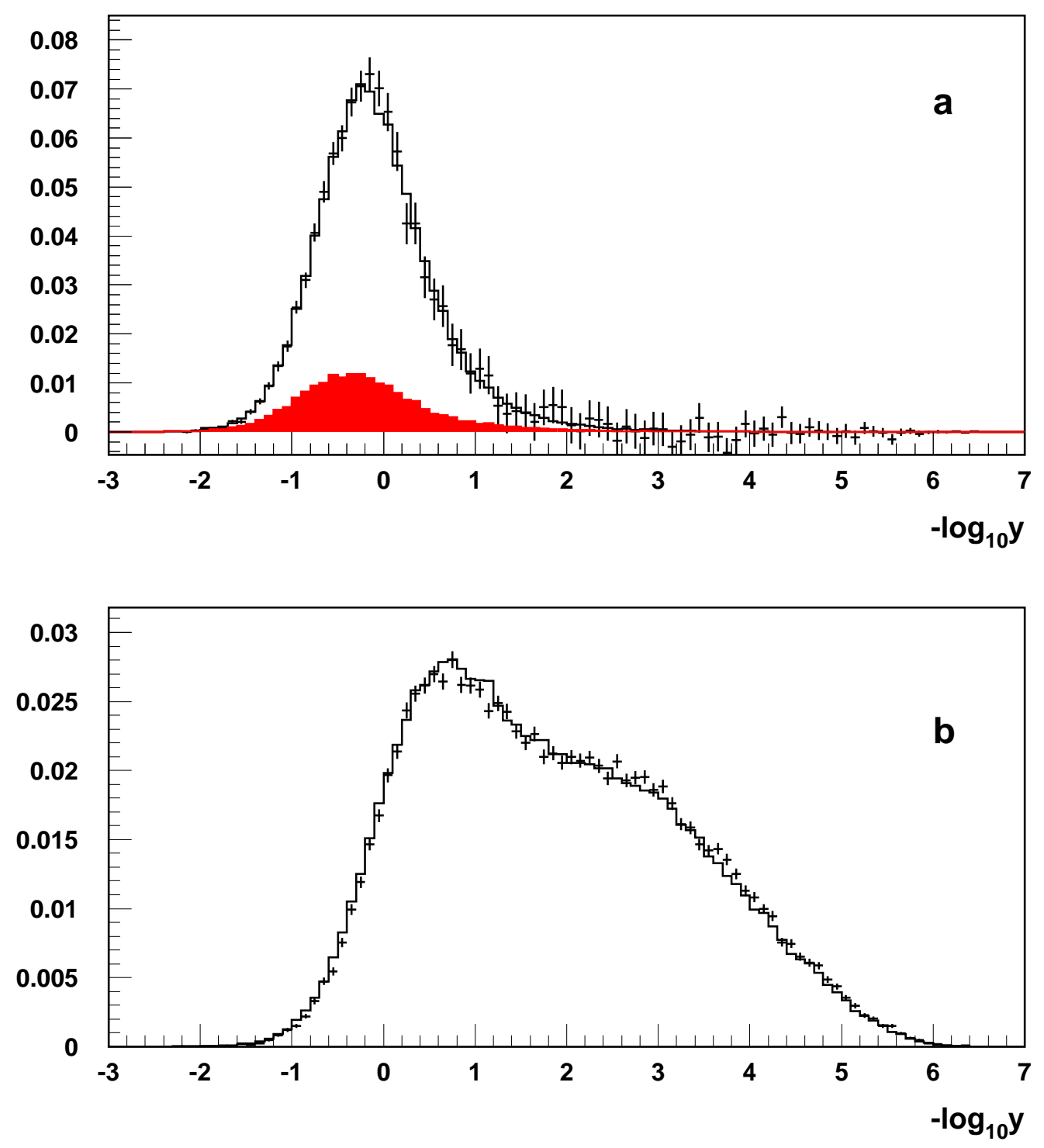

Figure 4: Distribution of the tagging variable $-\log _{10} y$ of the enhanced impact parameter method, for (a) background (u,d,s,c) jets and (b) jets with b-quarks. The points with errors are from the data and the histogram is the simulation prediction. The contribution of uds-quark jets is shown as the filled histogram in the upper figure. In (a), the data have been obtained by subtracting the b-enhanced distribution from the overall one; in (b) it is the b-enhanced data sample which is shown. 
of the better vertex finding efficiency and is almost zero for multiplicities less than two, where no vertex can be found. Since the invariant mass of the vertex is used in the tag, the number of neutrals in the $\mathrm{D}$ decay is also relevant. $\epsilon_{\mathrm{c}}$ drops strongly from zero to one neutrals, and significantly from one to more than one neutral in the $\mathrm{D}$ decay.

The evaluation of the uncertainty due to the charged decay multiplicity is detailed in [18]. For $\mathrm{D}^{0}$ and $\mathrm{D}^{+}$the relevant neutral multiplicities can be calculated from [9] to be:

$$
\begin{aligned}
\mathrm{BR}\left(\mathrm{D}^{0} \rightarrow \text { no neutrals }\right) & =(14.1 \pm 1.1) \% \\
\mathrm{BR}\left(\mathrm{D}^{0} \rightarrow 1 \text { neut. } \geq 2 \text { charged }\right) & =(37.7 \pm 1.7) \% \\
\mathrm{BR}\left(\mathrm{D}^{+} \rightarrow \text { no neutrals }\right) & =(11.2 \pm 0.6) \% \\
\mathrm{BR}\left(\mathrm{D}^{+} \rightarrow 1 \text { neut., } \geq 3 \text { charged }\right) & =(26.1 \pm 2.3) \%
\end{aligned}
$$

For the $\mathrm{D}_{\mathrm{s}}$ not enough information is available to calculate these branching fractions. However, it turns out that only $\mathrm{BR}\left(\mathrm{D}_{\mathrm{s}} \rightarrow K^{0} X\right)$ is relevant. Adding up the exclusive modes with and without $\mathrm{K}^{0}$ summarised in [9], a lower and upper limit of this branching ratio can be calculated from which $\mathrm{BR}\left(\mathrm{D}_{\mathrm{s}} \rightarrow K^{0} X\right)=(33 \pm 18) \%$ can be derived.

The largest physics contribution to the systematic error is the gluon splitting into a $\mathrm{b} \bar{b}$ pair. This quantity was recently measured [19], considerably reducing the systematic error due to this source and making it less dependent on theoretical assumptions [18].

To estimate the uncertainty on $\epsilon_{\mathrm{uds}}$ and $\epsilon_{\mathrm{c}}$ due to detector effects, four different methods were used:

- To estimate the effect of the track impact parameter's resolution, the parameterisation that describes the resolution of the detector [16] was changed within the uncertainty of its various coefficients. This corresponds to about $4 \%$ relative difference in the light and charm quark mistagging probabilities.

- Another test to estimate the effect of the detector resolution on $\epsilon_{\mathrm{c}}$ was the following: the resolution of the detector as estimated from the data was used in the definition of the tagging probability of simulated events. This second test was preferred for $\epsilon_{\mathrm{c}}$ because it is sensitive to systematic uncertainties related to the simulation of the charm background, since charmed particles have a detectable lifetime and a nonzero charged decay multiplicity. However it gave results consistent with the other method.

- To estimate the effect of correlations between tracks included in the probability $\left(P_{j}^{+}\right)$ calculation, the difference in tagging rate between data and simulation using tracks with negative impact parameters was taken as the uncertainty on $\epsilon_{\mathrm{uds}}$ (or by its statistical error if this was larger).

- The VD track efficiency in the simulation was varied by the amount of the residual difference between the data and the simulation (or by the statistical error on the difference is this was larger).

The errors obtained with the first, third and fourth tests were added in quadrature to obtain the final detector uncertainty on $\epsilon_{\mathrm{uds}}$. For $\epsilon_{\mathrm{c}}$ only the second and fourth tests were used.

\subsection{Hemisphere correlations}

In the extraction of $R_{\mathrm{b}}$, one has to correct for the fact that the two hemispheres in an event are not completely uncorrelated and thus the double tag efficiency, $\epsilon_{\mathrm{q}}^{(d)}$, is not exactly equal to the square of the hemisphere tagging efficiency. Due to the high purity, 


\begin{tabular}{|c|c|c|c|c|c|}
\hline \multirow[b]{2}{*}{ Source of systematic error } & \multirow[b]{2}{*}{ Range } & \multicolumn{2}{|c|}{ 1992-1993 } & \multicolumn{2}{|c|}{$1994-1995$} \\
\hline & & \begin{tabular}{|l|}
$\Delta \epsilon_{\text {uds }}$ \\
$\times 10^{5}$
\end{tabular} & $\begin{array}{c}\Delta \epsilon_{\mathrm{c}} \\
\times 10^{4}\end{array}$ & $\begin{array}{l}\Delta \epsilon_{\text {uds }} \\
\times 10^{5}\end{array}$ & $\begin{array}{c}\Delta \epsilon_{\mathrm{c}} \\
\times 10^{4}\end{array}$ \\
\hline MC statistics & & \pm 1.4 & \pm 0.7 & \pm 1.3 & \pm 0.7 \\
\hline Detector resolution & & \pm 1.3 & \pm 1.2 & \pm 3.3 & \pm 1.3 \\
\hline Detector efficiency & & \pm 1.0 & \pm 0.8 & \pm 1.0 & \pm 0.8 \\
\hline $\mathrm{K}^{0}$ & Tuned JETSET $\pm 10 \%$ & \pm 0.6 & & \pm 0.6 & \\
\hline Hyperons & Tuned JETSET $\pm 10 \%$ & \pm 0.2 & & \pm 0.1 & \\
\hline Photon conversions & $\pm 50 \%$ & \pm 1.0 & & \pm 0.4 & \\
\hline Gluon splitting $g \rightarrow \mathrm{b} \overline{\mathrm{b}}$ & $(0.269 \pm 0.067) \%$ & \pm 5.3 & \pm 0.5 & \pm 6.8 & \pm 0.7 \\
\hline Gluon splitting $g \rightarrow c \bar{c}$ & $(2.33 \pm 0.50) \%$ & \pm 1.2 & \pm 0.1 & \pm 2.3 & \pm 0.2 \\
\hline 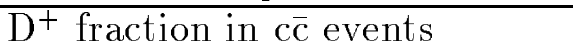 & $0.233 \pm 0.027^{1}$ & & \pm 0.8 & & \pm 1.0 \\
\hline $\mathrm{D}_{\mathrm{s}}$ fraction in $\mathrm{c} \overline{\mathrm{c}}$ events & $0.103 \pm 0.029^{1}$ & & $\mp 0.1$ & & $\mp 0.1$ \\
\hline c-baryon fraction in $c \bar{c}$ events & $0.063 \pm 0.028^{1}$ & & $\mp 0.9$ & & $\mp 0.9$ \\
\hline D decay multiplicity & see $[18]$ & & \pm 0.8 & & \pm 0.5 \\
\hline $\mathrm{BR}\left(\mathrm{D}^{0} \rightarrow\right.$ no neutrals $)$ & $(14.1 \pm 1.1) \%$ & & \pm 0.4 & & \pm 0.5 \\
\hline $\mathrm{BR}\left(\mathrm{D}^{0} \rightarrow 1\right.$ neut., $\geq 2$ charged $)$ & $(37.7 \pm 1.7) \%$ & & \pm 0.2 & & \pm 0.2 \\
\hline $\mathrm{BR}\left(\mathrm{D}^{+} \rightarrow\right.$ no neutrals $)$ & $(11.2 \pm 0.6) \%$ & & \pm 0.3 & & \pm 0.4 \\
\hline $\mathrm{BR}\left(\mathrm{D}^{+} \rightarrow 1\right.$ neut., $\geq 3$ charged $)$ & $(26.1 \pm 2.3) \%$ & & \pm 0.2 & & \pm 0.1 \\
\hline $\mathrm{BR}\left(\mathrm{D}_{\mathrm{s}} \rightarrow K^{0} X\right)$ & $(33 \pm 18) \%$ & & $\mp 0.8$ & & $\mp 1.0$ \\
\hline $\mathrm{D}^{0}$ lifetime & $0.415 \pm 0.004 \mathrm{ps}$ & & \pm 0.2 & & \pm 0.2 \\
\hline $\mathrm{D}^{+}$lifetime & $1.057 \pm 0.015 \mathrm{ps}$ & & \pm 0.2 & & \pm 0.2 \\
\hline $\mathrm{D}_{\mathrm{s}}$ lifetime & $0.447 \pm 0.017 \mathrm{ps}$ & & \pm 0.2 & & \pm 0.2 \\
\hline$\Lambda_{\mathrm{c}}$ lifetime & $0.206 \pm 0.012 \mathrm{ps}$ & & \pm 0.0 & & \pm 0.0 \\
\hline$\left\langle x_{E}(\mathrm{c})\right\rangle$ & $0.484 \pm 0.008$ & & \pm 0.3 & & \pm 0.4 \\
\hline \multicolumn{2}{|l|}{ Total c physics } & & \pm 1.8 & & \pm 2.0 \\
\hline \multicolumn{2}{|l|}{ Total } & \pm 6.0 & \pm 2.5 & \pm 8.1 & \pm 2.7 \\
\hline
\end{tabular}

Table 1: Systematic errors on the light and charm quark mistagging probabilities at the working point of $-\log _{10} y \geq 0.6$ for $1992-1993$ and $-\log _{10} y \geq 1.2$ for $1994-1995$, for the enhanced impact parameter method.

${ }^{1}$ Correlations between these sources are taken into account. 
this effect can safely be neglected for non-b events. For the 1993 (1994) data analysis ${ }^{3}$, it was estimated from the simulation to be $\rho=\frac{\epsilon_{\mathrm{b}}^{(d)}}{\epsilon_{\mathrm{b}}^{2}}-1=0.0342 \pm 0.0047(0.0198 \pm 0.0030)$ at the chosen working points.

Two main effects are responsible for $\rho$ not being equal to zero:

- Angular effects: the particles in an event are typically nearly back to back. This leads to a positive correlation due to the polar angle. The multiple scattering contribution to the VD resolution increases with decreasing polar angle and close to the end of the VD some tracks get lost outside its acceptance. There are also some minor effects connected with the azimuthal angle. Due to the flatness of the beam-spot at LEP, the resolution is better for horizontal than for vertical jets. Also, because of inefficient or poorly aligned modules, the detector is not completely homogeneous.

- QCD effects: Two effects contribute oppositely:

- Gluons emitted at large angles with respect to the quarks affect the energy of both quarks. As shown in figure 5 the b-tagging efficiency is a function of the momentum of the b-hadrons, leading to a positive correlation.

- In $2.2 \%$ of the events both b-quarks are boosted into the same hemisphere, recoiling against a hard gluon. This leads to a negative correlation. However, since the b-tagging efficiency for a hemisphere with two b's is about the same as for a hemisphere with only one b, this effect is suppressed.

To obtain the systematic error on the correlation estimate from the simulation, the fraction of events where both hemispheres are tagged was measured as a function of the relevant variable $\left(\cos \theta, \phi\right.$ or $\left.p_{j e t}\right)$ both in data and in simulation. From this, the contribution to the overall correlation due to that single variable can be estimated. This procedure uses the fact that the value of the test variable is correlated between the hemispheres, e.g. if one hemisphere has the cosine of its jet's polar angle at $\cos \theta$, the other one has its at $-\cos \theta$. The correlation coming from the polar angle can thus be calculated as

$$
\rho_{\theta}=\frac{2 \int_{0}^{z_{\max }} d z f(z) \epsilon_{\mathrm{b}}(z) \cdot \epsilon_{\mathrm{b}}(-z)}{\left(\int_{-z_{\max }}^{z_{\max }} d z f(z) \epsilon_{\mathrm{b}}(z)\right)^{2}}-1 \quad, z=\cos \theta .
$$

where $f(z)$ is the normalised angular distribution. For the angular variables all events have been used i.e. no attempt was made to select b events. Because of the high purity of the selected events and the fact that the initial polar and azimuthal angular distributions are identical for b- and light quark events, no bias was introduced. It was, however, verified that the conclusions did not change if a b-tag was required in the hemisphere opposite to the tested one. In all years a small difference $(\sim 0.15 \%)$ between data and the simulation has been found. Many tests have been carried out modifying the angular dependence of the b-tagging efficiency. Since the changes in the angular correlation always very closely followed the changes in the total correlation, the total correlation was corrected by half the difference between data and simulation; the systematic error was taken as the sum in quadrature of the full correction and the statistical error of the difference.

To investigate the correlation due to QCD effects, in all events the tracks were forced to be clustered as three jets, and the jet momenta were recalculated using energy-momentum conservation. The momentum of the fastest jet $\left(p_{j e t}\right)$ was then defined as the test variable

\footnotetext{
${ }^{3}$ In the following some results will be given only for the 1993 and 1994 data analyses, one for each microvertex setup.
} 


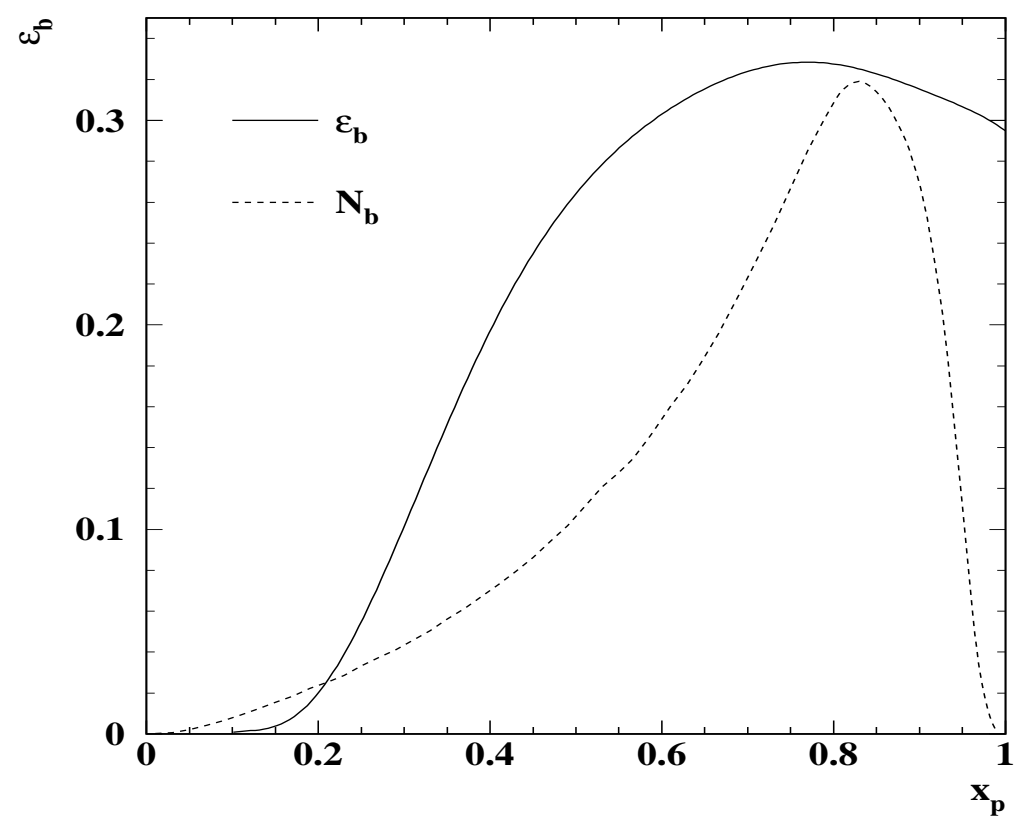

Figure 5: b-tagging efficiency as a function of the normalised b-hadron momentum (full curve), for the enhanced impact parameter method with the three-dimensional VD. At high $x_{p}$ the b-tagging efficiency drops slightly since, if the b-hadron is very energetic, only a few tracks are left to fit the primary vertex. The dashed curve shows the B-momentum spectrum with an arbitrary normalisation.

with the convention that it was assigned as positive in the one-jet hemisphere and negative in the two-jet hemisphere. Since the $p_{j e t}$ distribution is different for b and udsc events, a b-tag was required in the opposite hemisphere to avoid an artificial bias. As an additional complication, the two sources for QCD correlations act differently on the $p_{\text {jet }}$ distribution. If the two b-quarks are in opposite hemispheres, the one-jet hemisphere represents the faster and thus better tagged $\mathrm{b}$. If the two b-quarks are boosted into the same hemisphere, the one-jet side contains only a gluon. For that reason the one-jet hemisphere was only used if it passed a soft b-tag. On the two-jet side, a soft b-tag cannot be applied since this changes drastically the ratio of events with a fast $b$ and a soft gluon and vice versa. As systematic uncertainty the larger of the difference between the data and simulation measurements and the statistical error on this difference was taken. The systematic error induced by events with both b-quarks in one hemisphere was determined by varying their amount in simulation by $\pm 30 \%$, as suggested by a comparison of the JETSET parton shower and second order matrix element simulations.

Figure 6 shows the correlations for the different sources obtained with this procedure in data and simulation as a function of the b-tagging efficiency. Also shown for the simulation is the comparison between the sum of the different sources and the total correlation evaluated as $\rho=\frac{\epsilon_{\mathrm{b}}^{(d)}}{\epsilon_{\mathrm{b}}^{2}}-1$. The agreement of the sum of the different sources with the total correlation indicates that no important source has been forgotten.

Some additional physics systematics like B-lifetimes, decay multiplicities and fragmentation were also estimated by reweighting the simulation. The b-hadron decay multiplic- 
ity was recently measured $[20,18]$, considerably reducing its error. Because of the use of separate hemisphere primary vertices, the effects of these additional physics systematics were found to be small. The systematic errors on $R_{\mathrm{b}}$ arising from the uncertainties on $\rho$ are summarised in table 2.

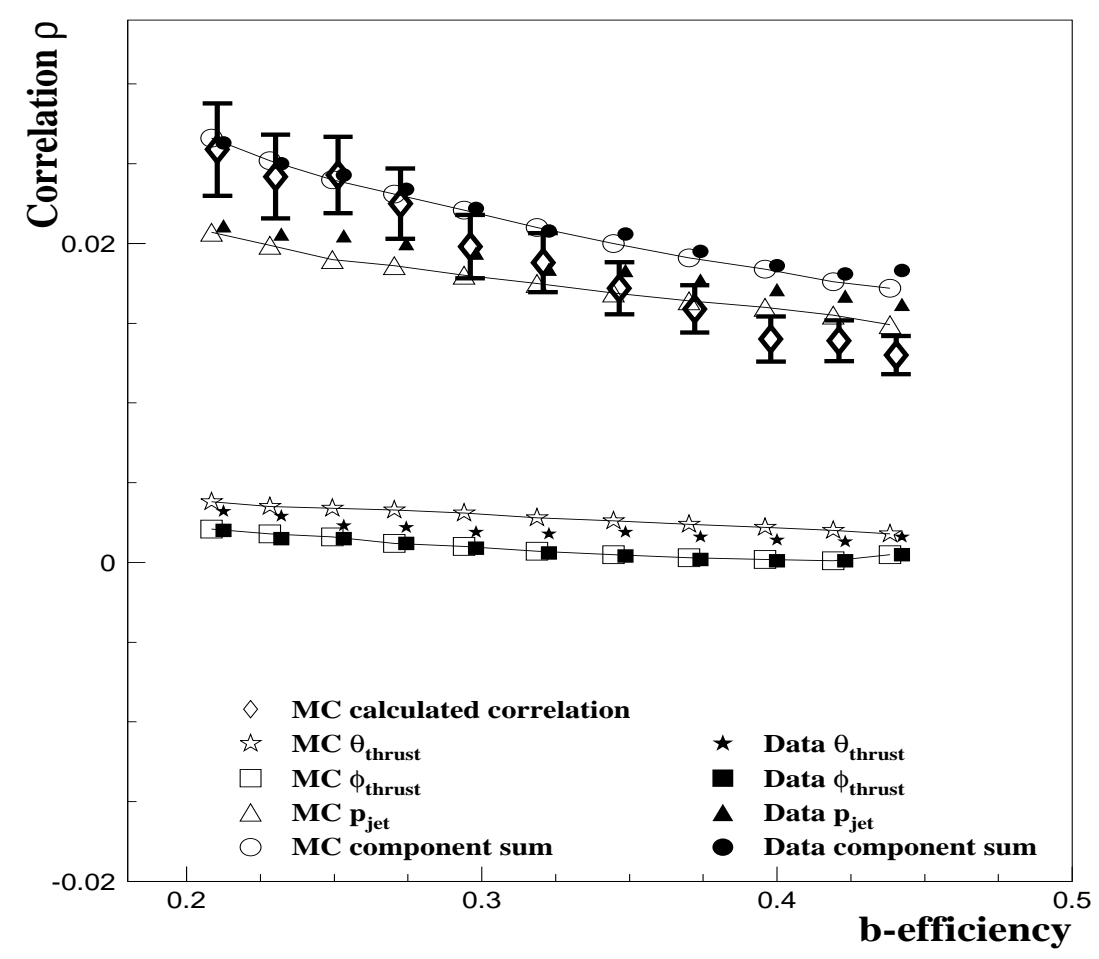

Figure 6: For the enhanced impact parameter method, the contributions to the hemisphere correlation due to the different sources and their sum as a function of the b-tagging efficiency are shown for the 1994 data and simulation. For the simulation the total correlation is also shown.

\begin{tabular}{||l|c|c|c||}
\hline & & \multicolumn{2}{|c||}{$\Delta R_{\mathrm{b}} \times 10^{4}$} \\
Source of systematics & Range & $1992-1993$ & $1994-1995$ \\
\hline MC statistics & & \pm 6.1 & \pm 3.8 \\
Two b-quarks in same hemisphere & $\pm 30 \%$ & \pm 2.1 & \pm 0.4 \\
$\left\langle x_{E}(\mathrm{~b})\right\rangle$ & $0.702 \pm 0.008$ & \pm 1.3 & \pm 0.6 \\
B decay multiplicity & $4.97 \pm 0.07$ & $\mp 1.7$ & $\mp 0.7$ \\
Average B lifetime & $1.55 \pm 0.05 \mathrm{ps}$ & $\mp 0.1$ & $\mp 0.0$ \\
\hline Angular effects & see text & \pm 3.4 & \pm 3.7 \\
Gluon radiation & see text & \pm 3.6 & \pm 2.6 \\
\hline \hline
\end{tabular}

Table 2: Systematic errors on $R_{\mathrm{b}}$ from hemisphere correlations, for the enhanced impact parameter analysis. 


\subsection{Results}

Table 3 summarises the number of hadronic $\mathrm{Z}$ decays selected in each year of operation, before and after the $\left|\cos \theta_{\text {thrust }}\right|$ cut. The numbers of single and double tagged events at the working points are also given.

\begin{tabular}{||c|c|c|c|c|c||}
\hline Year & 1992 & 1993 & 1994 & 1995 & Total \\
\hline Before $\left|\cos \theta_{\text {thrust }}\right|$ cut & 696,520 & 660,288 & $1,367,437$ & 664,493 & $3,388,738$ \\
After | cos $\theta_{\text {thrust }} \mid$ cut & 422,199 & 400,287 & 829,628 & 400,920 & $2,053,034$ \\
Single b-tags & 45,192 & 42,620 & 108,629 & 52,282 & 248,723 \\
Double b-tags & 5,503 & 5,158 & 16,078 & 7,784 & 34,523 \\
\hline
\end{tabular}

Table 3: Number of hadronic Z decays accepted for the analysis in each year of operation, before and after the $\left|\cos \theta_{\text {thrust }}\right|<0.65$ cut. The numbers of single and double tagged events are also given.

Using the above values of the mistagging probabilities and the correlation, with their errors, the measured values of $R_{\mathrm{b}}$ are:

$$
\begin{aligned}
& R_{\mathrm{b}}=0.21230 \pm 0.00211 \text { (stat) } \pm 0.00120 \text { (syst) }-0.026\left(R_{\mathrm{c}}-0.172\right) \\
& R_{\mathrm{b}}=0.21836 \pm 0.00224(\text { stat }) \pm 0.00113 \text { (syst) }-0.029\left(R_{\mathrm{c}}-0.172\right) \\
& R_{\mathrm{b}}=0.21772 \pm 0.00131 \text { (stat) } \pm 0.00076 \text { (syst) }-0.022\left(R_{\mathrm{c}}-0.172\right) \\
& R_{\mathrm{b}}=0.21653 \pm 0.00184 \text { (stat) } \pm 0.00109 \text { (syst) }-0.024\left(R_{\mathrm{c}}-0.172\right)
\end{aligned}
$$

where the first error is statistical and the second one systematic. The explicit dependence of these measurements on the assumed $R_{\mathrm{c}}$ value are also given. These results have also been corrected for $\tau$ background. The results for the four years are compatible and can be combined, with the following assumptions:

- all statistical errors are assumed to be independent,

- errors due to hemisphere correlations coming from gluon radiation are assumed to be fully correlated,

- systematic uncertainties due to angular effects were assumed correlated for 19941995 and also for 1992-1993, but uncorrelated between them owing to the independent microvertex configuration; the same was assumed for the detector effects on the estimate of light and charm quark mistagging probabilities,

- uncertainties due to uds, c and b physics simulation inputs were assumed to be fully correlated.

With these assumptions, the result for the full 1992-1995 data is:

$$
R_{\mathrm{b}}=0.21668 \pm 0.00088(\text { stat }) \pm 0.00070(\text { syst })-0.024\left(R_{\mathrm{c}}-0.172\right),
$$

where the $\chi^{2} /$ ndof of the combination is $4.5 / 3$. The mean b-purity at the working point for this measurement is $98.4 \%$.

The $\mathrm{b}$ hemisphere tagging efficiency was found to be $\epsilon_{\mathrm{b}}=0.2383 \pm 0.0025(0.2946 \pm$ 0.0018 ) for the 1993 (1994) data sample, compared to $\epsilon_{\mathrm{b}}(M C)=0.2300$ (0.2824) obtained from the simulation. The error is due only to the data statistics. In figure 7 a the ratio of the b-tagging efficiencies in 1994 data and simulation is given as a function of the 

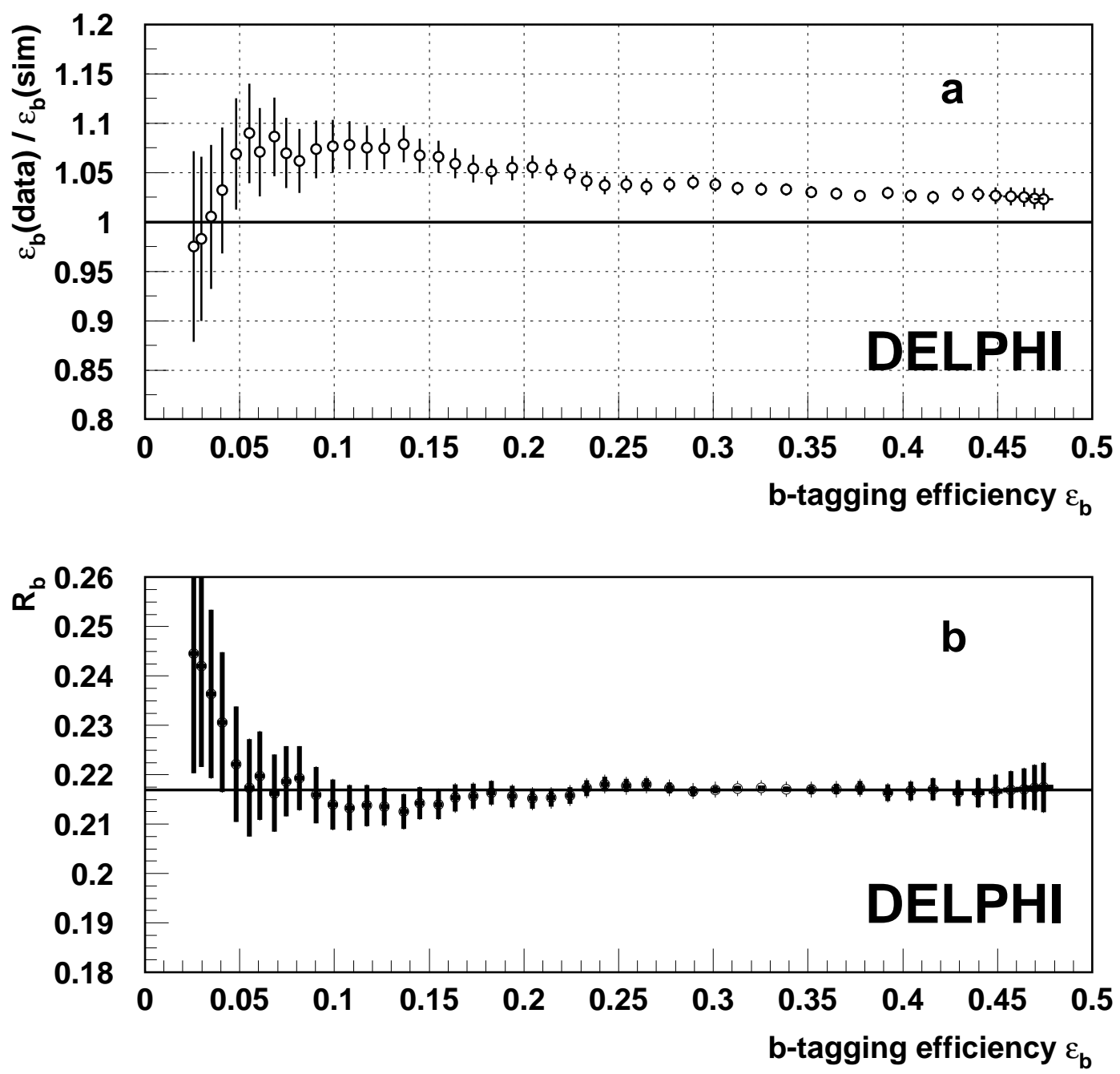

Figure 7: Enhanced impact parameter method. a) The ratio of the b-efficiency $\epsilon_{\mathrm{b}}$ measured in 1994 data and that taken from the simulation, as a function of the b-efficiency. b) The value of $R_{\mathrm{b}}$ with its total error as a function of the b-efficiency for 1994 data. The horizontal line corresponds to the value measured at the reference point, $-\log _{10} y \geq 1.2$, that corresponds to $\epsilon_{\mathrm{b}}=29.5 \%$. 
b-efficiency. The real data were about $4 \%$ more efficient than simulation. This difference can be explained by the uncertainties in the description of b-hadron production and decay.

A breakdown of the error for the chosen cuts of $-\log _{10} y$ is given in table 4 .

\begin{tabular}{||l|c|c|c|c|c||}
\hline & \multicolumn{5}{|c||}{$\Delta R_{\mathrm{b}} \times 10^{4}$} \\
Error Source & 1992 & 1993 & 1994 & 1995 & Comb. \\
\hline \hline Statistical error & \pm 21.1 & \pm 22.4 & \pm 13.1 & \pm 18.4 & \pm 8.8 \\
\hline Simulation statistics & \pm 9.3 & \pm 8.3 & \pm 4.4 & \pm 8.4 & \pm 3.4 \\
Light quark efficiency & \pm 2.8 & \pm 2.9 & \pm 3.1 & \pm 2.7 & \pm 2.8 \\
Charm efficiency & \pm 3.4 & \pm 3.6 & \pm 2.8 & \pm 3.1 & \pm 3.0 \\
Angular correlation & \pm 3.5 & \pm 3.7 & \pm 3.4 & \pm 4.3 & \pm 2.7 \\
Gluon radiation & \pm 3.6 & \pm 3.6 & \pm 2.6 & \pm 2.6 & \pm 2.9 \\
b physics correlation & \pm 3.0 & \pm 3.0 & \pm 1.0 & \pm 1.0 & \pm 1.7 \\
Acceptance bias & \pm 2.3 & \pm 1.8 & \pm 1.3 & \pm 2.2 & \pm 0.9 \\
\hline Total systematic error & \pm 12.0 & \pm 11.3 & \pm 7.6 & \pm 10.9 & \pm 7.0 \\
\hline \hline Total & \pm 24.3 & \pm 25.1 & \pm 15.1 & \pm 21.4 & \pm 11.2 \\
\hline
\end{tabular}

Table 4: Sources of error for the measurement of $R_{\mathrm{b}}$ using the enhanced impact parameter analysis, for all data sets and for the combined result.

As a cross-check of this measurement, a comparison of $R_{\mathrm{b}}$ as a function of the befficiency is given in figure $7 \mathrm{~b}$ for the 1994 data sample. The measured value of $R_{\mathrm{b}}$ is stable over a wide range of b-efficiency, and therefore of the purity and of the correlation.

\section{The Multivariate Analysis}

In the enhanced impact parameter analysis, hemispheres are tagged simply as $\mathrm{b}$ and non- $\mathrm{b}$. This leads to two independent tagging rates with six unknowns: $R_{\mathrm{b}}, \epsilon_{\mathrm{b}}, R_{\mathrm{c}}, \epsilon_{\mathrm{uds}}$, $\epsilon_{\mathrm{c}}$ and $\rho$. Three of them, $\rho$ and the mistag probabilities $\epsilon_{\mathrm{uds}}$ and $\epsilon_{\mathrm{c}}$, are then taken from simulation and $R_{\mathrm{c}}$ is fixed to the Standard Model value. If two or more tags are added the number of measurements increases faster than the number of additional tagging probabilities. In that way most of them can be determined from the data themselves. Furthermore, the fact that more hemispheres are accepted by the extra tags results in a smaller statistical error on $R_{\mathrm{b}}$.

\subsection{The method}

With some tagging algorithm, hemispheres of hadronic events containing $N_{F}=3$ flavours (uds, c and b) are classified into $N_{T}$ tagging categories or tags. The set of observables is then the matrix $f_{E}^{I J}$ with $I, J=1, \ldots, N_{T}$, defined as the observed fraction of events tagged as $I$ and $J$ for hemispheres 1 and 2 respectively. The corresponding expected fraction of events can be written as

$$
f_{E}^{I J}=\sum_{\mathrm{q}} \epsilon_{\mathrm{q}}^{I} \epsilon_{\mathrm{q}}^{J}\left(1+\rho_{\mathrm{q}}^{I J}\right) R_{\mathrm{q}} .
$$

In equation (7), $R_{\mathrm{q}}$ are the flavour fractions, satisfying $\sum_{\mathrm{q}} R_{\mathrm{q}}=1$, and $\epsilon_{\mathrm{q}}^{I}$ is the probability to classify a hemisphere of flavour $\mathrm{q}(=\mathrm{uds}, \mathrm{c}, \mathrm{b})$ as $\operatorname{tag} I$. The matrices $\rho_{\mathrm{q}}^{I J}$ account for 
hemisphere-hemisphere tagging correlations for flavour $\mathrm{q}$ and tags $I$ and $J$. All the hadronic hemispheres are classified as one of the tags, so that the conditions

$$
\sum_{I} \epsilon_{\mathrm{q}}^{I}=1, \quad \mathrm{q}=\mathrm{uds}, \mathrm{c}, \mathrm{b}
$$

and

$$
\sum_{I} \epsilon_{\mathrm{q}}^{I} \epsilon_{\mathrm{q}}^{J} \rho_{\mathrm{q}}^{I J}=0, \quad \mathrm{q}=\mathrm{uds}, \mathrm{c}, \mathrm{b} ; \quad J=1, \ldots, N_{T}
$$

must be satisfied. The $N_{T}\left(N_{T}+1\right) / 2-1$ independent measurements are therefore described by the following set of unknown independent parameters: $\left(N_{F}-1\right)$ flavour fractions, $N_{F}\left(N_{T}-1\right)$ tagging efficiencies and $N_{F} N_{T}\left(N_{T}-1\right) / 2$ correlation coefficients. The other correlation coefficients, arbitrarily chosen as $\rho_{\mathrm{q}}^{I N_{T}}$, are determined from the sum rules given in equations (8) and (9). As hemisphere correlations are kept small, the independent corrections $\rho_{\mathrm{q}}^{I J}$ for $I, J \neq N_{T}$ can be taken from simulation.

Due to an intrinsic ambiguity in the system, the full efficiency matrix cannot be determined from the data even if the number of measurements is larger than the number of unknowns [21]. If however $R_{\mathrm{c}}$ is fixed and the uds- and c-quark backgrounds for one tag (e.g. the b-tag with high purity, henceforth referred to as the b-tight tag) are taken from the simulation, all other efficiencies can be determined simultaneously with $R_{\mathrm{b}}$ by fitting the data. As detailed below, the enhanced impact parameter b-tag of the previous analysis is used to provide this tag. It has the largest effect on the analysis; all the other tags (two additional b-tags, one charm and one uds) provide additional constraints to improve the error and to cross-check the analysis. The dependences of $R_{\mathrm{b}}$ on these light quark contaminations are the same as in the enhanced impact parameter method and can be made small if the b-tight tag has high purity [22].

The systematic error reflects the uncertainties in the simulation calculations of the background mistag probabilities of the b-tight tag, $\epsilon_{\mathrm{uds}}^{\mathrm{b}-\text { tight }}$ and $\epsilon_{\mathrm{c}}^{\mathrm{b}-\text { tight }}$, and the correlations $\rho_{\mathrm{q}}^{I J}$ with $I, J \neq N_{T}$. The result is given as a function of the assumed value of $R_{\mathrm{c}}$. Even though the smallest number of tags to measure $R_{\mathrm{b}}$ with a constrained fit is now $N_{T}=4$, the choice $N_{T}=6$ was made in order to over-constrain the problem and to minimise the error. The number of independent observables is therefore 20 with 14 independent unknowns: 13 tagging efficiencies and $R_{\mathrm{b}}$.

\subsection{The hemisphere multiple tag}

To provide the six hemisphere tags, the enhanced impact parameter variable described in section 4 and defined by equation (2) is complemented by two additional flavour tagging algorithms. The tags are constructed in an attempt to isolate uds-, c- and b-quarks with high efficiency and purity, using exclusively the information provided by each hemisphere. In particular, in all the tagging methods the primary vertex is reconstructed independently in the two hemispheres, so hemisphere correlations are kept small.

The multivariate flavour tagging algorithm [22] is based on the large mass and relatively long lifetime of b-hadrons and some event shape properties of its decays. All the available information is combined using multivariate techniques. The lifetime information exploits the large impact parameters of tracks coming from B decays, together with a search for secondary vertices and their invariant masses. Finally, the lifetime information is combined with event shape properties of the $\mathrm{B}$ decays like large transverse momentum of the tracks with respect to the jet axis, rapidity distributions and the boosted sphericity. A total of $N=13$ variables is finally adopted. A detailed description of the variables is provided in reference [22]. 
The probabilities $p_{\mathrm{q}}^{\lambda}$ of observing a value of the variable $\lambda$ for a hemisphere of flavour $q$ are computed using model distributions taken from simulation. An estimate of the relative probability to observe simultaneously a set of $N$ variables is given by

$$
\mathcal{P}_{\mathrm{q}}=\frac{n_{\mathrm{q}} \prod_{\lambda=1}^{N} p_{\mathrm{q}}^{\lambda}}{\sum_{\mathrm{q}^{\prime}} n_{\mathrm{q}^{\prime}} \prod_{\lambda=1}^{N} p_{\mathrm{q}^{\prime}}^{\lambda}},
$$

where $n_{\mathrm{q}}=1$ for $\mathrm{q}=\mathrm{c}, \mathrm{b}$ and $n_{\mathrm{q}}=3$ for $\mathrm{q}=\mathrm{uds}$ hemispheres. The empirical factor 3 assigned to uds reflects the fact that this flavour is the sum of the three lighter flavours $\mathrm{u}, \mathrm{d}$ and $\mathrm{s}$, which are taken together because their distributions are similar. With this formulation the 5 flavours have the same weight.

In practice, what counts in comparing flavours are ratios of probabilities or differences of their logarithms. For this reason new estimators $\mathcal{L}_{\mathrm{q}}$, called flavour likelihoods, are introduced. $\mathcal{L}_{\mathrm{b}}$ is defined as

$$
\mathcal{L}_{\mathrm{b}}=\frac{2 \ln \mathcal{P}_{\mathrm{b}}-\ln \mathcal{P}_{\mathrm{uds}}-\ln \mathcal{P}_{\mathrm{c}}}{\sqrt{6}}
$$

and similarly for $\mathcal{L}_{\mathrm{u} d s}$ and $\mathcal{L}_{\mathrm{c}}$. A hemisphere can be classified according to the largest flavour likelihood (which is positive).

The flavour confidences method [23], similarly to the multivariate approach, is based not only on the track impact parameters but also on two other kinematic variables, the track momentum and the angle with respect to the jet axis. The method uses the simulation to build a function $\mathcal{C}_{\mathrm{q}}$ which gives the fraction of tracks coming from uds-, c- and b-quarks in a bin of three particle characteristics: impact parameter divided by its error, momentum and angle to the jet axis. Possible kinematic effects in the decay of b-hadrons produce correlations between the three quantities which are automatically taken into account by the three-dimensional binning. The individual flavour confidences are finally combined to construct the hemisphere tag:

$$
\mathcal{C} O N F_{\mathrm{q}}=\frac{n_{\mathrm{q}} \prod_{i} \mathcal{C}_{\mathrm{q}}^{i}}{\sum_{\mathrm{q}^{\prime}} n_{\mathrm{q}^{\prime}} \prod_{i} \mathcal{C}_{\mathrm{q}^{\prime}}^{i}},
$$

$\mathcal{C}_{\mathrm{q}}^{i}$ being the q flavour confidence for track $i$. As a cross-check, $R_{\mathrm{b}}$ has been measured using $\mathcal{C} O N F_{\mathrm{b}}$ as the only tag in the hemisphere [23]. For the data collected in 1992 through 1995, this yields

$$
R_{\mathrm{b}}=0.21855 \pm 0.00072(\text { stat }) \pm 0.00134 \text { (syst) }
$$

for $R_{\mathrm{c}}=0.172$.

Although some track information (in particular impact parameters, momentum and angle to the jet axis) is used in both methods, multivariate and confidences, it is used differently and the overlap is checked not to be complete. Thus interesting gains in performance can be obtained by a suitable mixture. Of the several methods of combination investigated, the one found to be the best was a simple linear combination for each flavour:

$$
\Delta_{\mathrm{q}}=(1-\alpha) \mathcal{L}_{\mathrm{q}}-\alpha \ln \left(1-\mathcal{C} O N F_{\mathrm{q}}\right)
$$

The quantities $\Delta_{\mathrm{q}}$ are called flavour multivariate discriminators and are the final basis of the classification. In principle, a different value of $\alpha$ could be used for each flavour, but it turns out that the same value $(\alpha=0.8)$ optimises the three flavours. The apparently high ratio $\alpha /(1-\alpha)=4$ is due to the fact that the range of values of the multivariate flavour 
likelihood is larger than that for the flavour confidences; it corresponds to approximately equal weights for the two components. Figure 8 shows the distributions of the flavour multivariate discriminators for data and simulation where the level of agreement can be seen over three orders of magnitude. The analysis is insensitive to small disagreements as they would affect only the tagging efficiencies, which are fitted from data. The effects on correlations are discussed in section 5.4.

The definition of the tags is given in table 5. Three of the six tags are designed to identify b-quarks, one c-quarks and one uds-quarks. Finally the no-tag category contains all hadronic hemispheres not classified in one of the previous tags, in order to satisfy the sum rules of equations (8) and (9). To avoid double counting, the hemisphere tags are defined in order of decreasing priority.

\begin{tabular}{||ccc|c|c||}
\hline Tag & Condition & Priority & \multicolumn{2}{|c||}{ Cut values } \\
& & & $1992-1993$ & $1994-1995$ \\
\hline b-tight & $y \leq y_{0}$ & 6 & 0.6 & 1.2 \\
b-standard & $\Delta_{\mathrm{b}} \geq \Delta_{\mathrm{b}, 0}^{\text {high }}$ & 5 & 3.5 & 3.5 \\
b-loose & $\Delta_{\mathrm{b}} \geq \Delta_{\mathrm{b}, 0}^{l o w}$ & 4 & 1.4 & 1.2 \\
charm & $\Delta_{\mathrm{c}} \geq \Delta_{\mathrm{c}, 0}$ & 3 & 0.58 & 0.65 \\
uds & $\Delta_{\mathrm{uds}} \geq \Delta_{\mathrm{uds}, 0}$ & 2 & 2.7 & 3.2 \\
no-tag & & 1 & & \\
\hline
\end{tabular}

Table 5: The hemisphere tags defined in order of decreasing priority.

The b-tight tag has the strongest influence on $R_{\mathrm{b}}$ and the cut $-\log _{10} y_{0}$ was fixed at 1.2 in 1994-1995 and 0.6 in 1992-1993 to minimise the total error. All other cuts were chosen in order to obtain good efficiencies with reasonable backgrounds in the relevant tags. They are given in table 5. The simulation expectations for the efficiencies are given separately for 1993 and 1994 in table 6 . This table is a measurement of the performance of the tags and tagging techniques all together. In this analysis of $R_{\mathrm{b}}$, only the charm and light quark backgrounds of the b-tight tag are taken from simulation. Therefore the light and charm quark systematic errors of the enhanced impact parameter analysis are valid for this measurement of $R_{\mathrm{b}}$. All the other efficiencies are measured directly from the data and can be used as a cross-check of the analysis (see table 6 and table 8 ).

\begin{tabular}{||c|ccc|ccc||}
\hline & \multicolumn{3}{|c|}{1993} & \multicolumn{3}{c||}{1994} \\
Tag $I$ & $\epsilon_{\mathrm{uds}}^{I}$ & $\epsilon_{\mathrm{c}}^{I}$ & $\epsilon_{\mathrm{b}}^{I}$ & $\epsilon_{\mathrm{uds}}^{I}$ & $\epsilon_{\mathrm{c}}^{I}$ & $\epsilon_{\mathrm{b}}^{I}$ \\
\hline b-tight & 0.00050 & 0.00381 & 0.23003 & 0.00052 & 0.00376 & 0.28236 \\
b-standard & 0.00188 & 0.02631 & 0.17051 & 0.00126 & 0.02692 & 0.15578 \\
b-loose & 0.01446 & 0.07754 & 0.16043 & 0.01219 & 0.07858 & 0.15158 \\
charm & 0.05814 & 0.16428 & 0.05704 & 0.04942 & 0.15617 & 0.04963 \\
uds & 0.11977 & 0.03579 & 0.00548 & 0.11819 & 0.03025 & 0.00471 \\
no-tag & 0.80530 & 0.69226 & 0.37649 & 0.81856 & 0.70431 & 0.35591 \\
\hline
\end{tabular}

Table 6: Simulation results for the tagging efficiencies at the nominal cuts for 1993 and 1994 for the multivariate analysis.

Compared with the enhanced impact parameter analysis in which only b-tight tagged hemispheres are used, in the multivariate analysis all hadronic hemispheres are tagged, allowing the statistical accuracy to be increased. As will be shown in section 5.5, the systematic uncertainty on $R_{\mathrm{b}}$ due to hemisphere correlations is also improved. 

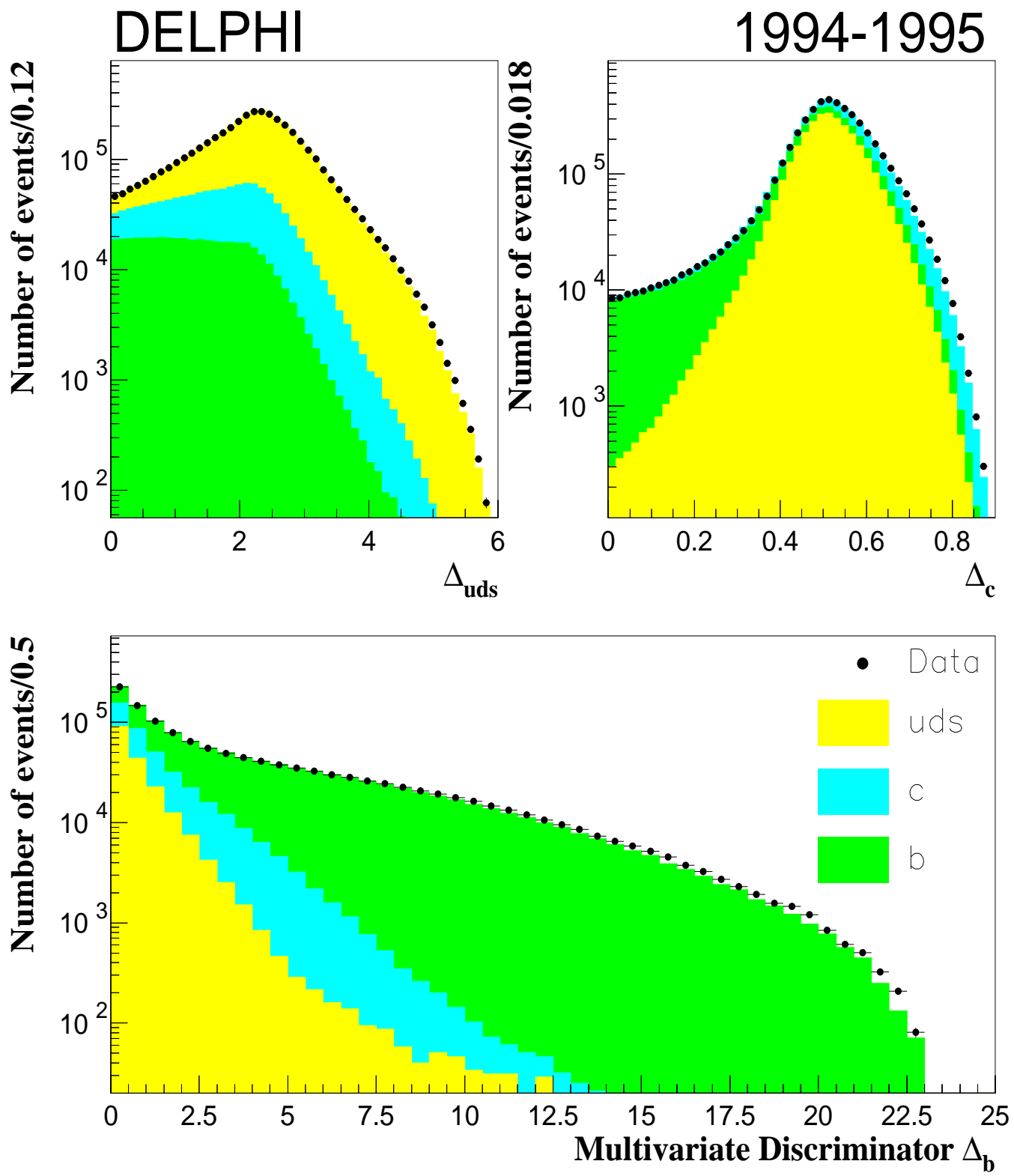

Figure 8: Distribution of the multivariate discriminator $\Delta_{\mathrm{q}}$ in the uds-, c- and b-tags for 1994-1995 data and simulation. The different types of shading show the different flavour contributions to the simulated event sample. The simulation distributions are normalised to the data statistics. 


\subsection{The measurement of $R_{\mathrm{b}}$}

The experimentally measured numbers for the different categories of doubly tagged events which passed the $\left|\cos \theta_{\text {thrust }}\right|$ cut are given in table 7 for the 1993 and 1994 analyses.

\begin{tabular}{||c|cccccc||}
\hline & \multicolumn{7}{|c||}{1993} \\
Tag & b-tight & b-standard & b-loose & charm & uds & no-tag \\
\hline b-tight & 5,158 & & & & & \\
b-standard & 7,405 & 2,762 & & & & \\
b-loose & 6,839 & 5,070 & 2,764 & & & \\
charm & 2,568 & 2,388 & 4,196 & 4,026 & & \\
uds & 268 & 416 & 1,408 & 5,504 & 4,068 & \\
no-tag & 15,224 & 14,204 & 22,719 & 47,804 & 51,151 & 194,345 \\
\hline \hline & \multicolumn{7}{|c}{1994} & & \\
Tag & b-tight & b-standard & b-loose & charm & uds & no-tag \\
\hline b-tight & 16,078 & \multicolumn{7}{c}{} & \\
b-standard & 17,049 & 4,564 & & & & \\
b-loose & 16,261 & 9,017 & 5,025 & & & \\
charm & 5,737 & 4,150 & 7,386 & 6,757 & & \\
uds & 662 & 766 & 2,583 & 9,877 & 9,210 & \\
no-tag & 36,764 & 25,527 & 43,749 & 88,319 & 109,031 & 411,116 \\
\hline
\end{tabular}

Table 7: For the multivariate analysis, measured numbers of doubly tagged events passing the $\left|\cos \theta_{\text {thrust }}\right|$ cut in 1993 and 1994.

The fit of $R_{\mathrm{b}}$ and the efficiencies to these numbers gives the following results for each year of operation:

$$
\begin{array}{lll}
R_{\mathrm{b}}=0.21278 \pm 0.00164(\text { stat }), & \chi^{2} / \text { ndof }=8.5 / 6 & (1992), \\
R_{\mathrm{b}}=0.21759 \pm 0.00171(\text { stat }), & \chi^{2} / \text { ndof }=7.0 / 6 & (1993), \\
R_{\mathrm{b}}=0.21689 \pm 0.00099(\text { stat }), & \chi^{2} / \text { ndof }=7.6 / 6 & (1994), \\
R_{\mathrm{b}}=0.21601 \pm 0.00145(\text { stat }), & \chi^{2} / \text { ndof }=3.4 / 6 & (1995)
\end{array}
$$

The errors are only statistical. The efficiencies obtained from the same fits for 1993 and 1994 are shown in table 8 . They can be compared with the simulation predictions of table 6. For a complete comparison, an estimate of the systematic errors must be included. The good values of $\chi^{2} /$ ndof for all years indicate consistency between the different tags and show especially that the $R_{\mathrm{b}}$ values obtained with the enhanced impact parameter analysis and the multivariate analysis are consistent within their statistical errors.

\subsection{Systematic errors}

Systematic errors are due to the quantities estimated from simulation: event selection bias, light and charm quark backgrounds in the b-tight tag and hemisphere correlations.

\subsubsection{Uncertainties in light and charm quark mistag probabilities}

The sensitivities $s$ of $R_{\mathrm{b}}$ to light and charm quark mistag probability uncertainties are the same as in the enhanced impact parameter analysis. The sensitivity to background 


\begin{tabular}{||c|ccc||}
\hline & \multicolumn{4}{|c||}{1993} \\
Tag $I$ & $\epsilon_{\text {uds }}^{I}$ & $\epsilon_{\mathrm{c}}^{I}$ & $\epsilon_{\mathrm{b}}^{I}$ \\
\hline b-tight & 0.00050 & 0.00381 & $0.2388 \pm 0.0017$ \\
b-standard & $0.0025 \pm 0.0005$ & $0.0236 \pm 0.0025$ & $0.1746 \pm 0.0011$ \\
b-loose & $0.0135 \pm 0.0007$ & $0.0806 \pm 0.0035$ & $0.1604 \pm 0.0012$ \\
charm & $0.0730 \pm 0.0008$ & $0.1799 \pm 0.0025$ & $0.0575 \pm 0.0010$ \\
uds & $0.1266 \pm 0.0009$ & $0.0332 \pm 0.0034$ & $0.0052 \pm 0.0005$ \\
no-tag & $0.7839 \pm 0.0017$ & $0.6790 \pm 0.0063$ & $0.3636 \pm 0.0021$ \\
\hline \hline & \multicolumn{4}{|c}{1994} \\
Tag $I$ & $\epsilon_{\text {uds }}^{I}$ & $\epsilon_{\mathrm{c}}^{I}$ & $\epsilon_{\mathrm{b}}^{I}$ \\
\hline b-tight & 0.00052 & 0.00376 & $0.2959 \pm 0.0012$ \\
b-standard & $0.0019 \pm 0.0003$ & $0.0239 \pm 0.0014$ & $0.1574 \pm 0.0007$ \\
b-loose & $0.0124 \pm 0.0004$ & $0.0788 \pm 0.0020$ & $0.1493 \pm 0.0008$ \\
charm & $0.0614 \pm 0.0005$ & $0.1692 \pm 0.0015$ & $0.0512 \pm 0.0006$ \\
uds & $0.1291 \pm 0.0005$ & $0.0310 \pm 0.0017$ & $0.0050 \pm 0.0002$ \\
no-tag & $0.7947 \pm 0.0008$ & $0.6933 \pm 0.0034$ & $0.3412 \pm 0.0013$ \\
\hline
\end{tabular}

Table 8: Tagging efficiencies with statistical errors for data as measured from the multivariate fit at the nominal cuts for 1993 and 1994. For a complete comparison of the fit results with the simulation, an estimate of the systematic errors must be included.

is defined as the relative change of $R_{\mathrm{b}}$ due to the change of the background mistag probability,

$$
\frac{\Delta R_{\mathrm{b}}}{R_{\mathrm{b}}}=s_{\mathrm{q}} \frac{\Delta \epsilon_{\mathrm{q}}^{\mathrm{b}-\text { tight }}}{\epsilon_{\mathrm{b}}^{\mathrm{b}-\text { tight }}},
$$

In this analysis, $s_{\text {uds }}=-5.7$ and $s_{\mathrm{c}}=-1.5$ for light and charm quarks respectively. The systematic uncertainties on $\epsilon_{\mathrm{uds}}^{\mathrm{b}-\mathrm{tight}}$ and $\epsilon_{\mathrm{c}}^{\mathrm{b}-\text { tight }}$ have been estimated in section 4.4, where table 1 shows the breakdown into the different sources.

\subsubsection{Hemisphere correlation uncertainties}

The $\rho_{\mathrm{q}}^{I J}$ hemisphere correlation coefficients as estimated from the simulation for the 1994 analysis, together with their sensitivities to $R_{\mathrm{b}}$ are given in the second column of table 9 , where the errors are due to simulation statistics. Only the relevant correlations with a sensitivity higher than 0.010 are shown. The sensitivity $s$ is defined as the relative change on $R_{\mathrm{b}}$ due to a change of a given correlation, $\frac{\Delta R_{\mathrm{b}}}{R_{\mathrm{b}}}=s \Delta \rho_{\mathrm{q}}^{I J}$. The sensitivity of this measurement of $R_{\mathrm{b}}$ to $\rho_{\mathrm{b}}^{\mathrm{b}-\text { tight,b-tight }}$ is 0.767 for $1994-1995$ and 0.693 for $1992-1993$, compared to unity in the enhanced impact parameter analysis. However, as shown in the table, there are other correlations with important sensitivities which have zero sensitivity in the enhanced impact parameter analysis. As explained in section 5.1, correlations containing the no-tag category ( $I$ or $J=N_{T}$, which have a complex mixture of flavours being statistically significant) are determined from the sum rules, so they have a negligible sensitivity on the analysis.

Systematic errors on $\rho_{\mathrm{q}}^{I J}$ arise from uncertainties in the simulation both of uds, $\mathrm{c}$ and $\mathrm{b}$ physics and of the vertex detector acceptance and gluon radiation. The latter can be estimated by isolating the contributions to correlations and comparing their effect in data and simulation. The variables used to isolate the correlation sources are exactly the same as described in section 4: the polar and azimuthal angles and $p_{j e t}$. 


\begin{tabular}{|c|c|c|c|c|c|c|c|c|c|c|}
\hline & \multirow{2}{*}{\multicolumn{2}{|c|}{ MC global Sensitivity }} & \multicolumn{2}{|r|}{$\cos \theta_{\text {thrust }}$} & \multicolumn{3}{|c|}{$\phi_{\text {thrust }}$} & \multicolumn{3}{|c|}{$p_{\text {jet }}$} \\
\hline & & & $\mathrm{MC}$ & $\mathrm{RD}$ & $\mathrm{MC}$ & $\mathrm{RD}$ & $\bar{D}$ & $\mathrm{MC}$ & $\mathrm{RD}$ & $\mathrm{D}$ \\
\hline \multicolumn{11}{|c|}{ b correlations } \\
\hline$\rho_{\mathrm{b}}^{\mathrm{b}-\text { tight,b-tight }}$ & $0.0198 \pm 0.0020$ & 0.767 & 0.0031 & 0.00190 .0002 & 0.0010 & 0.0009 & 0.0009 & 0.0180 & 0.0194 & 0.0007 \\
\hline$\rho_{\mathrm{b}}^{\mathrm{b}-\mathrm{tight}, \mathrm{b}-\mathrm{standard}}$ & $0.0034 \pm 0.0020$ & 0.219 & 0.0013 & $-0.0003 \quad 0.0003$ & -0.0008 & -0.0009 & 0.0010 & 0.0184 & 0.0195 & 0.0005 \\
\hline$\rho_{\mathrm{b}}^{\mathrm{b}-\text { tight }, \mathrm{b}-\text { loose }}$ & $0.0031 \pm 0.0020$ & 0.107 & 0.0004 & -0.00020 .0002 & -0.0017 & -0.0014 & 0.0003 & 0.0098 & 0.0104 & 0.0005 \\
\hline$\rho_{\mathrm{b}}^{\mathrm{b}-\text { tight,charm }}$ & $0.0047 \pm 0.0039$ & -0.041 & -0.0042 & $-0.0026 \quad 0.0025$ & 0.0027 & 0.0009 & 0.0004 & -0.0175 & -0.0179 & 0.0012 \\
\hline$\rho_{\mathrm{b}}^{\mathrm{b}-\text { standard, } \mathrm{b}-\text { standard }}$ & $0.0073 \pm 0.0037$ & -0.081 & 0.0032 & $0.0035 \quad 0.0005$ & 0.0003 & -0.0006 & 0.0005 & 0.0183 & 0.0179 & 0.0010 \\
\hline$\rho_{\mathrm{b}}^{\mathrm{b}-\text { standard,b-loose }}$ & $0.0034 \pm 0.0031$ & -0.088 & 0.0033 & 0.00330 .0004 & 0.0006 & -0.0016 & 0.0008 & 0.0106 & 0.0118 & 0.0006 \\
\hline$\rho_{\mathrm{b}}^{\mathrm{b}-\text { standard,charm }}$ & $0.0042 \pm 0.0058$ & 0.023 & -0.0129 & $-0.0115 \quad 0.0010$ & -0.0004 & -0.0001 & 0.0001 & -0.0104 & -0.0082 & 0.0013 \\
\hline$\rho_{\mathrm{b}}^{\mathrm{b}-\text { loose }, \mathrm{b}-\text { loose }}$ & $0.0095 \pm 0.0038$ & -0.047 & 0.0038 & 0.00290 .0005 & 0.0012 & -0.0012 & 0.0010 & 0.0082 & 0.0085 & 0.0007 \\
\hline$\rho_{\mathrm{b}}^{\mathrm{b}-\text { loose,charm }}$ & $-0.0079 \pm 0.0059$ & 0.014 & -0.0137 & -0.01120 .0009 & -0.0011 & -0.0001 & 0.0001 & -0.0242 & -0.0272 & 0.0011 \\
\hline \multicolumn{11}{|c|}{ c correlations } \\
\hline$\rho_{\mathrm{c}}^{\mathrm{b}-\text { standard,charm }}$ & $0.0015 \pm 0.0173$ & 0.014 & -0.0100 & $-0.0078 \quad 0.0003$ & 0.0018 & 0.0014 & 0.0003 & 0.0133 & 0.0088 & 0.0007 \\
\hline$\rho_{\mathrm{c}}^{\mathrm{b}-\text { loose,charm }}$ & $0.0028 \pm 0.0097$ & 0.024 & -0.0135 & $-0.0106 \quad 0.0003$ & 0.0005 & 0.0001 & 0.0001 & 0.0146 & 0.0167 & 0.0011 \\
\hline$\rho_{\mathrm{c}}^{\text {charm,charm }}$ & $0.0434 \pm 0.0080$ & -0.013 & 0.0183 & 0.00950 .0002 & 0.0021 & -0.0001 & 0.0001 & 0.0117 & 0.0168 & 0.0007 \\
\hline \multicolumn{11}{|c|}{ uds correlations } \\
\hline \begin{tabular}{|l} 
charm,uds \\
chds
\end{tabular} & $0.0134 \pm 0.0078$ & 0.020 & 0.0093 & 0.00920 .0006 & -0.0012 & -0.0009 & 0.0002 & 0.0182 & 0.0174 & 0.0007 \\
\hline \begin{tabular}{||l|l} 
uds,uds \\
uds
\end{tabular} & $0.0758 \pm 0.0057$ & 0.034 & 0.0079 & $0.0091 \quad 0.0007$ & 0.0048 & 0.0042 & 0.0004 & 0.0383 & 0.0339 & 0.0012 \\
\hline
\end{tabular}

Table 9: b, c and uds correlations of the multivariate analysis, with major sensitivity $(>0.010)$ on $R_{\mathrm{b}}$ at the nominal cuts for the 1994 data. The individual contributions to the total correlation for the data (RD) and for the simulation (MC) are shown, together with the statistical error on their difference (D). 


\begin{tabular}{||l|c|c|c||}
\hline & & \multicolumn{2}{|c||}{$\Delta R_{\mathrm{b}} \times 10^{4}$} \\
Source of systematics & Range & $1992-1993$ & $1994-1995$ \\
\hline MC statistics & & \pm 5.6 & \pm 3.8 \\
Two b-quarks in same hemisphere & $\pm 30 \%$ & \pm 0.7 & \pm 0.4 \\
$\left\langle x_{E}(\mathrm{~b})\right\rangle$ & $0.702 \pm 0.008$ & \pm 1.8 & \pm 1.0 \\
$\mathrm{~B}$ decay multiplicity & $4.97 \pm 0.07$ & $\mp 1.5$ & $\mp 0.7$ \\
Average B lifetime & $1.55 \pm 0.05 \mathrm{ps}$ & $\mp 0.0$ & $\mp 0.0$ \\
Gluon splitting $g \rightarrow \mathrm{c} \overline{\mathrm{c}}$ & $(2.33 \pm 0.50) \%$ & \pm 0.1 & \pm 0.1 \\
Gluon splitting $g \rightarrow \mathrm{b} \overline{\mathrm{b}}$ & $(0.269 \pm 0.067) \%$ & $\mp 0.1$ & $\mp 0.1$ \\
Charm physics & see table 1 & \pm 0.7 & \pm 0.4 \\
\hline Angular effects & & \pm 2.7 & \pm 0.7 \\
Gluon radiation & & \pm 1.7 & \pm 2.5 \\
\hline \hline Total & & \pm 6.9 & \pm 4.8 \\
\hline
\end{tabular}

Table 10: Systematic errors due to hemisphere correlations for the multivariate analysis.

The contribution to $\rho_{\mathrm{q}}^{I J}$ from one of the above variables $z$ can be determined through the following expression:

$$
\rho_{\mathrm{q}, z}^{I J}=\frac{\int d z f_{\mathrm{q}}(z)\left[\epsilon_{\mathrm{q}}^{I, \text { same }}(z) \epsilon_{\mathrm{q}}^{J, \text { oppo }}(z)+\epsilon_{\mathrm{q}}^{J, \text { same }}(z) \epsilon_{\mathrm{q}}^{I, \text { oppo }}(z)\right]}{2\left[\int d z f_{\mathrm{q}}(z) \epsilon_{\mathrm{q}}^{I, \text { same }}(z)\right]\left[\int d z f_{\mathrm{q}}(z) \epsilon_{\mathrm{q}}^{J, \text { same }}(z)\right]}-1,
$$

where $f_{\mathrm{q}}(z)$ is the distribution of the variable $z$ for the flavour $\mathrm{q}$, and $\epsilon_{\mathrm{q}}^{I, \text { same }}(z)$ and $\epsilon_{\mathrm{q}}^{I, \text { oppo }}(z)$ are the efficiencies to tag a hemisphere of flavour $\mathrm{q}$ as a function of $z$ in the same and opposite hemisphere respectively.

The contribution $\rho_{\mathrm{q}, z}^{I J}$ can easily be computed for the simulation where the flavour $\mathrm{q}$ is known. However, comparison of data and simulation requires the experimental isolation of this flavour in the data. This flavour isolation is obtained successfully for uds- and b-quarks using a soft multivariate tag, but not for c-quarks owing to the small charm event statistics and the rather poor c-quark purity. However, the quoted systematic uncertainties are not affected because of the small sensitivity of $R_{\mathrm{b}}$ to c correlations. The $\mathrm{b}$ and uds selections are achieved by imposing the cuts $\Delta_{\mathrm{b}}>3.3(3.0)$ and $\Delta_{\mathrm{uds}}>2.9(3.3)$ in 1992-1993 (1994-1995) on the opposite hemisphere to the tested one. These cuts are chosen to achieve hemisphere b- and uds-purities of about $92 \%$. In addition, as described in section 4.5, to reduce the effect of two b-quarks boosted into the same hemisphere, when testing the correlation due to QCD effects, the one-jet hemisphere is only used if it passed a soft multivariate tag of purity about $76 \%\left(\Delta_{\mathrm{b}}>0.9\right.$ in $1992-1993$ and $\Delta_{\mathrm{b}}>0.6$ in 1994-1995). Table 9 gives the results of this procedure for all relevant correlation coefficients in 1994, for data and simulation. Figure 6 shows the total correlation as a function of the b-tight tag efficiency, together with each of the three components and their sum as obtained with this procedure, for simulation and data.

The systematic errors for the QCD and the angular correlations are finally estimated essentially as in the enhanced impact parameter analysis. To determine the effect of the discrepancies between simulation and data as seen a given column in table 9 , the change in $R_{\mathrm{b}}$ is found when all the correlations are changed from their simulated values to the data ones. The errors from these sources are combined quadratically to give the final error (see table 10). 
The effect of the uncertainties in the physical parameters used in the simulation of correlations is determined by varying these physics inputs within their experimental ranges around their central values, according to the prescription given in reference [18]. For each variation of these physical parameters, obtained by reweighting the simulation, all the correlation correction factors are recomputed, allowing a new determination of $R_{\mathrm{b}}$. The change observed in $R_{\mathrm{b}}$ is assigned as the systematic error from this source. Table 10 summarises the errors on $R_{\mathrm{b}}$ due to these physical uncertainties. Since parameters like the $\mathrm{B}$ decay multiplicity or the hadron lifetimes are a priori uncorrelated between the two hemispheres, they can only influence the correlations in an indirect way by modifying the sensitivity to angular or QCD effects. That means that these sources are already largely accounted for in the data-simulation tests. However since these uncertainties are small but potentially correlated with other experiments, they are conservatively taken into account. The correlation between uds and charm efficiency uncertainties and hemisphere-hemisphere correlations due to some of these physics inputs is small enough that it can be neglected.

\subsection{Results and consistency checks}

From all previous numbers, the final results for each data sample are the following:

$$
\begin{aligned}
& R_{\mathrm{b}}=0.21278 \pm 0.00164(\text { stat }) \pm 0.00107 \text { (syst) }-0.026\left(R_{\mathrm{c}}-0.172\right) \\
& R_{\mathrm{b}}=0.21759 \pm 0.00171 \text { (stat) } \pm 0.00098(\text { syst })-0.029\left(R_{\mathrm{c}}-0.172\right) \\
& R_{\mathrm{b}}=0.21689 \pm 0.00099 \text { (stat) } \pm 0.00068 \text { (syst) }-0.022\left(R_{\mathrm{c}}-0.172\right) \\
& R_{\mathrm{b}}=0.21601 \pm 0.00145 \text { (stat) } \pm 0.00099 \text { (syst) }-0.024\left(R_{\mathrm{c}}-0.172\right)
\end{aligned}
$$

These results should be compared with the enhanced impact parameter analysis results of section 4.6. They are compatible when taking into account uncorrelated errors, as indicated by the good values of $\chi^{2} /$ ndof of section 5.3 .

The results for the four years are compatible and can be combined, with the same assumptions as detailed in section 4.6. The result for the full 1992-1995 data is:

$$
R_{\mathrm{b}}=0.21616 \pm 0.00067 \text { (stat) } \pm 0.00060(\text { syst })-0.024\left(R_{\mathrm{c}}-0.172\right),
$$

where the $\chi^{2} /$ ndof of the combination is $4.2 / 3$. As previously, the mean b-purity at the working point for this measurement is $98.4 \%$.

Figures 9 and 10 show the stability of the final $R_{\mathrm{b}}$ result as a function of the b-tight tag efficiency for the 1994-1995 and 1992-1993 combinations respectively, together with the contributions to the total error. It can be seen that the minimum error is obtained at a b-efficiency of $29.6 \%$ (i.e. for a cut $-\log _{10} y \geq 1.2$ ) in $1994-1995$, and of $27.1 \%$ (cut $-\log _{10} y \geq 0.4$ ) in $1992-1993$. However, to have similar purities in all years and to minimise the combined error, the cut $-\log _{10} y \geq 0.6$ was taken for 1992-1993, which corresponds to a b-efficiency of $23.9 \%$.

Figure 11 shows the stability of $R_{\mathrm{b}}$ as a function of all other hemisphere tag efficiencies (i.e. b-standard, b-loose, charm and uds) for 1994-1995.

Table 11 shows a breakdown of the error on this measurement. From the direct comparison of this table with table 4, it can be seen that both statistical errors and systematic uncertainties coming from hemisphere correlations are improved. 


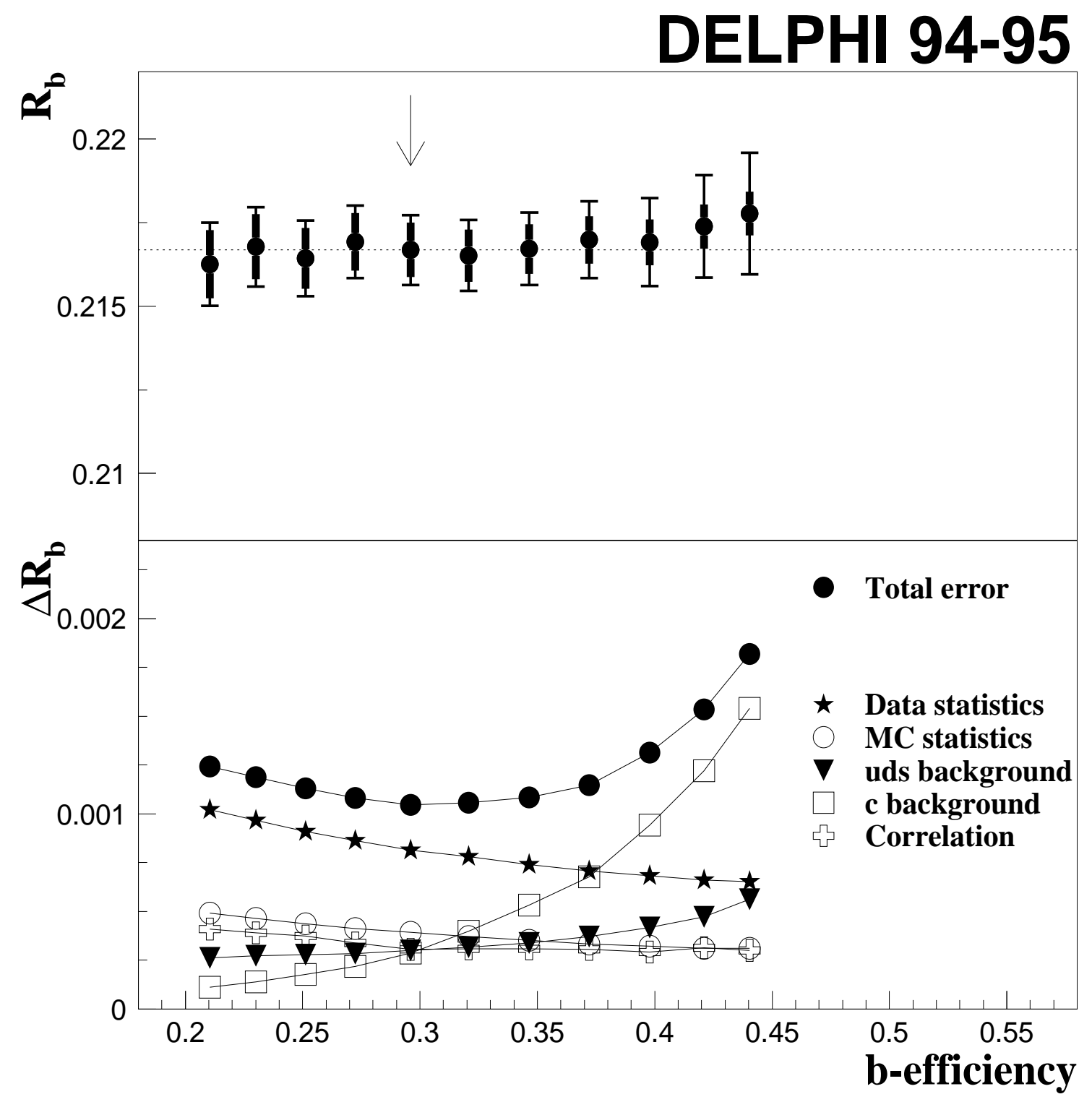

Figure 9: Stability of the 1994-1995 multivariate $R_{\mathrm{b}}$ result as a function of the b-tight tag efficiency, together with the contributions to the total error. The minimum error is obtained at an efficiency of $29.6 \%$, where the b-purity is $98.5 \%$. In the upper plot the thick error bar represents the statistical uncertainty and the narrow one is the total error. All errors are correlated from point to point. The arrow marks the position of the working point and the dotted line shows the value at that cut. 


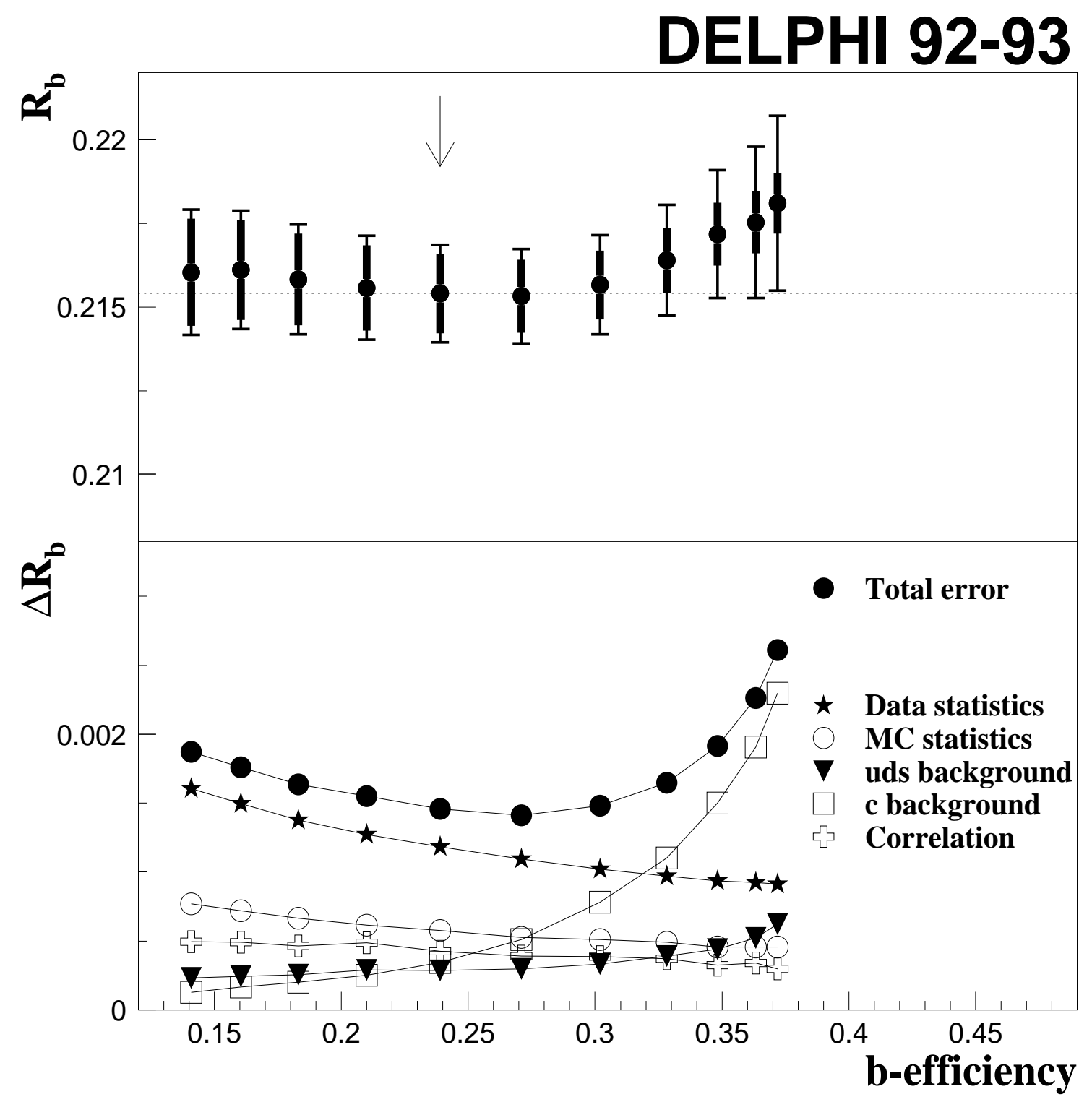

Figure 10: Stability of the 1992-1993 multivariate $R_{\mathrm{b}}$ result as a function of the b-tight tag efficiency, together with the contributions to the total error. The working point is chosen to have a similar purity to that at the working point of the 1994-1995 analysis. It results in an efficiency of $23.9 \%$ with a b-purity of $98.2 \%$. All errors are correlated from point to point. The arrow marks the position of the working point and the dotted line shows the value at that cut. 

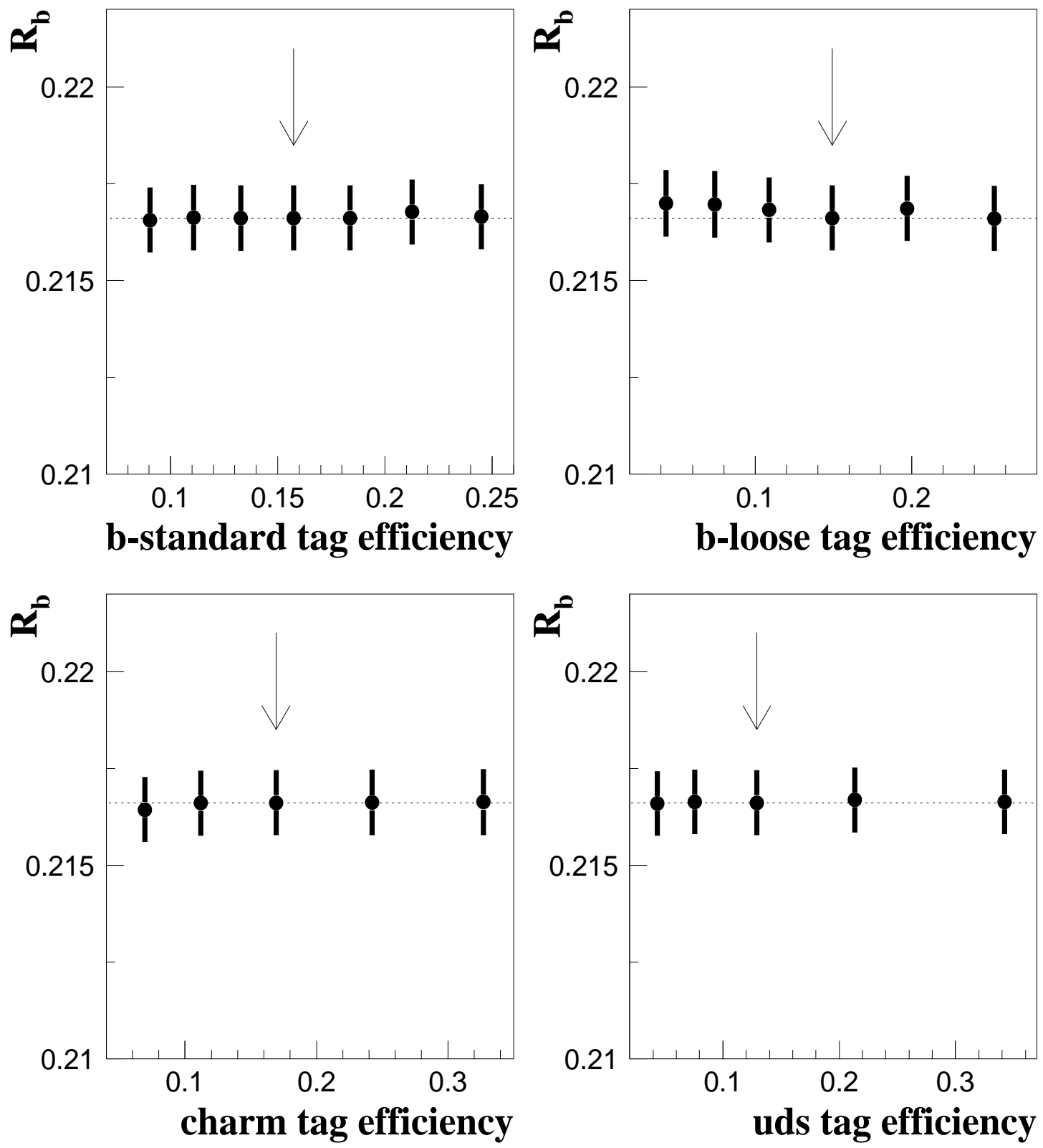

Figure 11: Stability of the multivariate $R_{\mathrm{b}}$ result as a function of the efficiencies of the b-standard, b-loose, charm and uds hemisphere tags for 1994-1995. Only the statistical errors are shown. Errors are correlated from point to point. The arrow marks the position of the working point and the dotted line shows the value at that cut. 


\begin{tabular}{||l|c|c|c|c|c||}
\hline & \multicolumn{5}{|c||}{$\Delta R_{\mathrm{b}} \times 10^{4}$} \\
Error Source & 1992 & 1993 & 1994 & 1995 & Comb. \\
\hline \hline Statistical error & \pm 16.4 & \pm 17.1 & \pm 9.9 & \pm 14.5 & \pm 6.7 \\
\hline Simulation statistics & \pm 8.7 & \pm 7.6 & \pm 4.4 & \pm 8.2 & \pm 3.3 \\
uds mistag prob & \pm 2.8 & \pm 2.9 & \pm 3.1 & \pm 2.7 & \pm 2.8 \\
Charm mistag prob & \pm 3.4 & \pm 3.6 & \pm 2.8 & \pm 3.1 & \pm 3.0 \\
Angular correlation & \pm 2.7 & \pm 2.7 & \pm 0.6 & \pm 1.5 & \pm 0.9 \\
Gluon radiation & \pm 1.7 & \pm 1.7 & \pm 2.5 & \pm 2.5 & \pm 2.2 \\
Physics correlation & \pm 2.5 & \pm 2.5 & \pm 1.4 & \pm 1.4 & \pm 1.7 \\
Acceptance bias & \pm 2.3 & \pm 1.8 & \pm 1.3 & \pm 2.2 & \pm 0.9 \\
\hline Total systematic error & \pm 10.7 & \pm 9.8 & \pm 6.8 & \pm 9.9 & \pm 6.0 \\
\hline \hline Total & \pm 19.6 & \pm 19.7 & \pm 12.0 & \pm 17.6 & \pm 9.0 \\
\hline
\end{tabular}

Table 11: Sources of error for the measurement of $R_{\mathrm{b}}$ using the multivariate analysis, for all data sets and for the combined result.

\section{Secondary Vertex Analysis}

An independent analysis was carried out on the 1992-1995 data sets using only reconstructed vertex information for the tagging of b-quarks. The hadronic event selection and the impact parameter fine tuning were in common with the enhanced impact parameter and multivariate analyses.

\subsection{Secondary vertex search}

The search for secondary vertices was made independently inside event hemispheres defined by the plane perpendicular to the event thrust axis. Hemisphere tracks used in the analysis were required to fulfil the following criteria to ensure precise tracks:

- $R \phi$ hits in at least 2 layers of the VD,

- an impact parameter in the $R \phi$ plane with respect to the beam-spot of less than $0.15 \mathrm{~cm}$,

- a momentum greater than $750 \mathrm{MeV} / c$.

In addition $\mathrm{K}_{S}^{0}$ and $\Lambda$ particles and photon conversions were reconstructed, details of which are given in [11]. Tracks coming from the decay of a $\mathrm{K}_{S}^{0}$ or $\Lambda$ or from a photon conversion were rejected.

Candidate secondary vertices were identified by the following procedure:

- All possible three-track vertices in the $R \phi$ plane were found. Candidates were rejected if any of the following conditions was met: i) the decay length, $L$, to the beam-spot was smaller than $\sigma_{L}$, where $\sigma_{L}$ is the uncertainty on $L$; ii) $L>3.0 \mathrm{~cm}$; and iii) the $\chi^{2}$ probability for forming a vertex out of these tracks, $P\left(\chi^{2}\right)$, was less than $1 \%$.

- Next, an attempt was made to add to candidate vertices any track likely to have originated from the same point in space. Each track falling within a cone of half-angle 0.4 radian placed around the candidate vertex momentum vector was fitted in turn to the vertex. That track which contributed the largest increase in $L / \sigma_{L}$ was added permanently to the candidate vertex provided that: i) $L>3 \sigma_{L}$; ii) $L<3.0 \mathrm{~cm}$; and iii) $P\left(\chi^{2}\right)>1 \%$. This procedure was continued until no more tracks could be added. 
- In an attempt to identify cascade decays, $b \rightarrow c$, where the $b$ and $c$ vertices are significantly separated, further tracks were added to the vertex if they were consistent, within the errors, with the candidate while at the same time inconsistent with the beam-spot.

- Finally, using a procedure similar to that outlined above, a single primary vertex per hemisphere was found from tracks that were consistent with the beam-spot position. A unique track was defined to be one that was included in a candidate secondary vertex but was not part of the primary vertex. Secondary vertex candidates that did not contain a unique track were removed.

Close attention was paid to reducing light quark backgrounds, which are most sensitive to the modelling of the tracking in the simulation. For the case of vertices containing two unique tracks that included $z$-hits, these two tracks were separately fitted to form a threedimensional vertex point. Requiring $P\left(\chi^{2}\right)>0.1 \%$ was found to be an effective cut for removing cases where badly reconstructed tracks might form a vertex in two dimensions but were clearly unassociated with each other once the $z$-coordinate was considered. Note that this procedure was only possible for data taken with the three-dimensional VD, i.e. from 1994 onwards.

If after the secondary vertex finding procedure there was more than one candidate vertex in a hemisphere, the vertex containing the largest number of unique tracks was chosen to tag the hemisphere.

\subsection{Tagging $\mathrm{Z} \rightarrow \mathrm{b} \overline{\mathrm{b}}$ events}

In order to tag $Z \rightarrow b \bar{b}$ events, the output of a neural network [24] was used with five input variables derived from the properties of the reconstructed vertices. The neural network was trained using $5000 \mathrm{~b} \overline{\mathrm{b}}$ and $5000(\mathrm{u} \overline{\mathrm{u}}+\mathrm{d} \overline{\mathrm{d}}+\mathrm{s} \overline{\mathrm{s}}+\mathrm{c} \overline{\mathrm{c}})$ simulated events.

The input variables were

1. the number of unique tracks in the secondary vertex,

2. the number of tracks in the primary vertex that were not also associated to any secondary,

3. the number of tracks in common to both the secondary and primary vertices,

4. the decay length significance $L / \sigma_{L}$,

5 . the secondary vertex rapidity, defined as

$$
R=\ln \frac{E+P_{\|}}{\sqrt{m_{0}^{2}+P_{t}^{2}}},
$$

where $E$ is the energy, $m_{0}$ the invariant mass assuming the pion mass for all the particles, $P_{t}$ the absolute value of the summed transverse momenta and $P_{\|}$the summed longitudinal momenta of unique tracks in the vertex with respect to the jet axis.

Distributions of all input variables and the resulting neural network output for simulation and data are shown in figure 12 .

The calculation of $R_{\mathrm{b}}$ followed the single/double hemisphere method described in section 4 . 

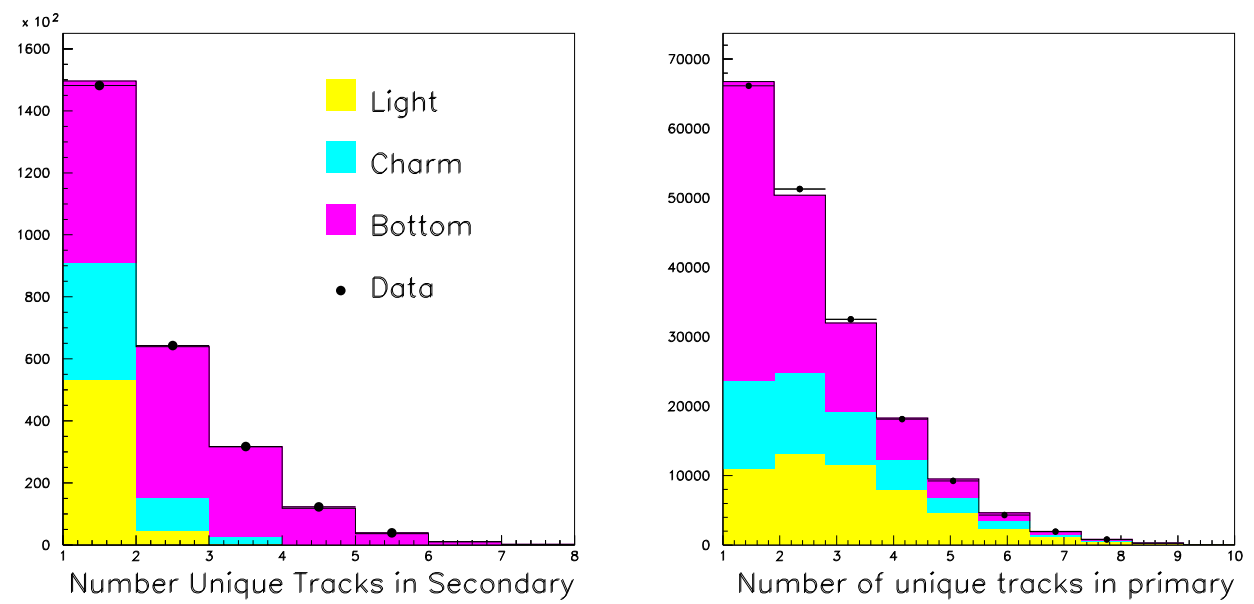

\section{DELPHI}
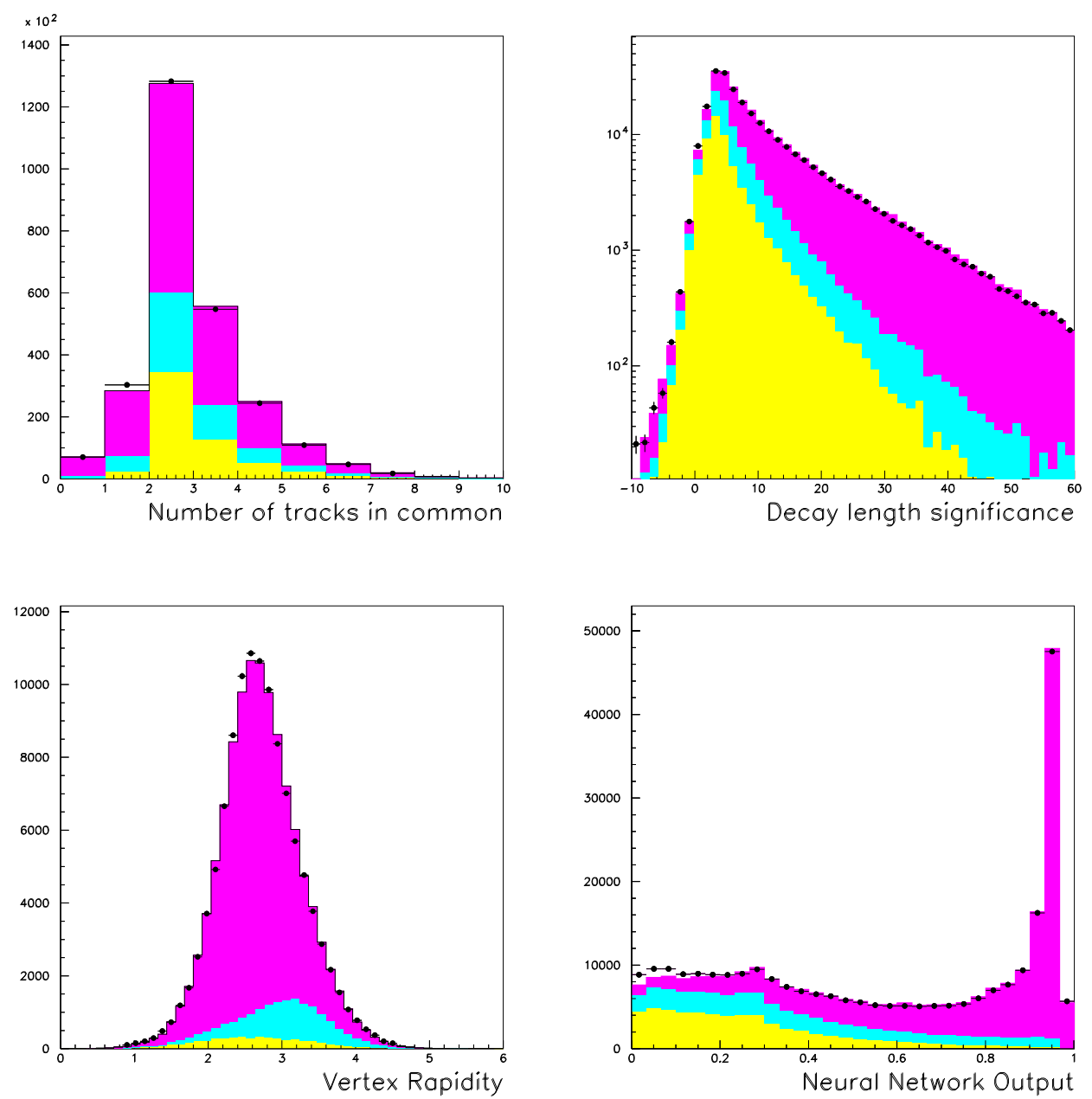

Figure 12: For the secondary vertex analysis, distributions comparing data and simulation for the 5 input variables to the neural network and for the neural network output itself. 


\subsection{Quantities from the simulation}

The various sources of systematic error are listed in table 12. Both the light and charm quark mistag probabilities as well as the hemisphere correlation were extracted from the simulation. The uncertainty due to detector effects was estimated in a similar way to that described in section 4.4. It should be noted that the simulation sample used to train the neural network was excluded from that used in the determination of the light quark mistagging probabilities.

\begin{tabular}{|c|c|c|c|c|c|}
\hline \multirow[b]{2}{*}{ Source of systematic error } & \multirow[b]{2}{*}{ Range } & \multicolumn{2}{|c|}{$1992-1993$} & \multicolumn{2}{|c|}{ 1994-1995 } \\
\hline & & $\begin{array}{l}\Delta \epsilon_{\text {uds }} \\
\times 10^{5}\end{array}$ & $\begin{array}{c}\Delta \epsilon_{\mathrm{c}} \\
\times 10^{4}\end{array}$ & $\begin{array}{l}\Delta \epsilon_{\text {uds }} \\
\times 10^{5}\end{array}$ & $\begin{array}{c}\Delta \epsilon_{\mathrm{c}} \\
\times 10^{4}\end{array}$ \\
\hline MC statistics & & \pm 2.2 & \pm 1.3 & \pm 1.9 & \pm 1.3 \\
\hline Detector resolution & & \pm 2.2 & \pm 3.1 & \pm 1.9 & \pm 2.7 \\
\hline Light hadron modelling & Tuned JETSET $\pm 10 \%$ & \pm 4.2 & & \pm 5.0 & \\
\hline Gluon splitting $g \rightarrow \mathrm{b} \overline{\mathrm{b}}$ & $(0.269 \pm 0.067) \%$ & \pm 5.8 & & \pm 6.0 & \\
\hline Gluon splitting $g \rightarrow c \bar{c}$ & $(2.33 \pm 0.50) \%$ & \pm 3.4 & & \pm 3.4 & \\
\hline $\mathrm{D}^{+}$fraction in ce events & $0.233 \pm 0.027^{1}$ & & \pm 4.0 & & \pm 3.7 \\
\hline $\mathrm{D}_{\mathrm{s}}$ fraction in $\mathrm{c} \overline{\mathrm{c}}$ events & $0.103 \pm 0.029^{1}$ & & $\mp 0.7$ & & $\mp 0.5$ \\
\hline c-baryon fraction in $c \bar{c}$ events & $0.063 \pm 0.028^{1}$ & & $\mp 2.7$ & & $\mp 2.3$ \\
\hline D decay multiplicity & see $[18]$ & & \pm 3.5 & & \pm 3.1 \\
\hline $\mathrm{BR}\left(\mathrm{D}^{0} \rightarrow\right.$ no neutrals $)$ & $(14.1 \pm 1.1) \%$ & & \pm 1.4 & & \pm 1.3 \\
\hline $\mathrm{BR}\left(\mathrm{D}^{0} \rightarrow 1\right.$ neut., $\geq 2$ charged $)$ & $(37.7 \pm 1.7) \%$ & & \pm 0.3 & & \pm 0.3 \\
\hline $\mathrm{BR}\left(\mathrm{D}^{+} \rightarrow\right.$ no neutrals $)$ & $(11.2 \pm 0.6) \%$ & & \pm 0.7 & & \pm 0.7 \\
\hline $\mathrm{BR}\left(\mathrm{D}^{+} \rightarrow 1\right.$ neut., $\geq 2$ charged $)$ & $(26.1 \pm 2.3) \%$ & & \pm 1.8 & & \pm 1.6 \\
\hline $\mathrm{BR}\left(\mathrm{D}_{\mathrm{s}} \rightarrow K^{0} X\right)$ & $(33 \pm 18) \%$ & & \pm 1.0 & & \pm 0.6 \\
\hline $\mathrm{D}^{0}$ lifetime & $0.415 \pm 0.004 \mathrm{ps}$ & & \pm 1.0 & & \pm 0.9 \\
\hline $\mathrm{D}^{+}$lifetime & $1.057 \pm 0.015 \mathrm{ps}$ & & \pm 0.6 & & \pm 0.6 \\
\hline $\mathrm{D}_{\mathrm{s}}$ lifetime & $0.447 \pm 0.017 \mathrm{ps}$ & & \pm 0.6 & & \pm 0.6 \\
\hline$\Lambda_{\mathrm{c}}$ lifetime & $0.206 \pm 0.012 \mathrm{ps}$ & & \pm 0.9 & & \pm 0.4 \\
\hline$\left\langle x_{E}(\mathrm{c})\right\rangle$ & $0.484 \pm 0.008$ & & \pm 2.1 & & \pm 2.7 \\
\hline \multicolumn{2}{|l|}{ Total c physics } & & \pm 7.1 & & \pm 6.6 \\
\hline \multicolumn{2}{|l|}{ Total } & \pm 8.5 & \pm 7.8 & \pm 8.9 & \pm 7.2 \\
\hline
\end{tabular}

Table 12: Contributions to the systematic uncertainties on the mistagging probabilities for light and charm quarks in the secondary vertex analysis, as estimated from the simulation.

The various sources of systematic error are shown in the lower half of figure 13, together with the statistical and total errors, as a function of the efficiency for identifying b-quarks in the 1994 data set. The arrow in the upper half indicates the working point where the minimum total error is found. At this point, taking 1994 data as an example, the b-purity in the simulation was $94.8 \%$ for a b-tagging efficiency of $26.4 \%$, to be compared with an efficiency of $(28.2 \pm 0.2) \%$ calculated from the data. The same cut on the neural network output was used for each data set and resulted in similar b-tagging performances.

The light and charm quark mistag probabilities extracted from the 1994 simulation at the working point were:

$$
\epsilon_{\mathrm{uds}}=(0.100 \pm 0.009) \times 10^{-2}
$$




$$
\epsilon_{\mathrm{c}}=(1.35 \pm 0.08) \times 10^{-2}
$$

Their contributions to the systematic error on $R_{\mathrm{b}}$ are summarised in table 13 year by year, and also on the combined results.

Correlations between hemispheres come from both geometrical and kinematic effects, as described in section 4.5. The hemisphere correlation in b events for this analysis was estimated from the 1994 simulation to be

$$
\rho=(0.53 \pm 0.21(\text { stat }) \pm 0.08(\text { syst })) \times 10^{-2},
$$

with similar values obtained for the other data sets. The first error is due to the limited simulation statistics and the second is the estimated systematic uncertainty. Contributions to the systematic error from both geometrical effects and physics modelling, for each of the years, are estimated as described in section 4.5 and the resulting uncertainties on the measurement of $R_{\mathrm{b}}$ are summarised in table 13 .

\subsection{Results}

Using the numbers of data events with one and with both hemispheres tagged and the values for the mistagging probabilities and correlations of which examples are given above, and taking into account the selection bias towards $\mathrm{Z} \rightarrow \mathrm{b} \overline{\mathrm{b}}$ events, $R_{\mathrm{b}}$ was calculated separately for each data set to be:

$$
\begin{aligned}
& R_{\mathrm{b}}=0.21746 \pm 0.00192 \text { (stat) } \pm 0.00150 \text { (syst) }-0.093\left(R_{\mathrm{c}}-0.172\right) \\
& R_{\mathrm{b}}=0.21830 \pm 0.00189 \text { (stat) } \pm 0.00138(\text { syst })-0.089\left(R_{\mathrm{c}}-0.172\right) \\
& R_{\mathrm{b}}=0.21609 \pm 0.00138 \text { (stat) } \pm 0.00120 \text { (syst) }-0.087\left(R_{\mathrm{c}}-0.172\right) \\
& R_{\mathrm{b}}=0.21835 \pm 0.00221 \text { (stat) } \pm 0.00153 \text { (syst) }-0.082\left(R_{\mathrm{c}}-0.172\right)
\end{aligned}
$$

The stability of the measurement over a range of b-tagging efficiencies can be seen in the upper half of figure 13, again taking 1994 as an example.

The results for the four years are compatible and can be combined with the same assumptions as detailed in section 4.6, to give a final value for the 1992-1995 data sets of

$$
R_{\mathrm{b}}=0.21722 \pm 0.00089 \text { (stat) } \pm 0.00112 \text { (syst) }-0.088\left(R_{\mathrm{c}}-0.172\right) .
$$

The full breakdown of the uncertainties on the combined result is given in table 13 .

\section{Energy Dependence}

In 1993 and 1995, data were taken at three different centre-of-mass energies $(\sqrt{s}=$ $89.46,91.27,93.00 \mathrm{GeV})$. As the relative contributions of photon exchange and $\gamma-\mathrm{Z}$ interference are strongly suppressed at energies close to the $\mathrm{Z}$ resonance, $R_{\mathrm{b}}(\sqrt{s})$ is predicted to be almost constant in the Standard Model. However, if $R_{\mathrm{b}}$ is affected by the interference of the $\mathrm{Z}$ with another particle like a $\mathrm{Z}^{\prime}[25]$ which is almost degenerate in mass, some energy dependence can be expected if the mass and width of the $\mathrm{Z}^{\prime}$ are not exactly equal to those of the Z. Similar effects could arise from an R-parity violating sneutrino [26].

Since the b-tagging efficiency varies only very little within the energy range considered here, no complicated single to double tag comparison is needed to measure $\frac{R_{\mathrm{b}}(\sqrt{s})}{R_{\mathrm{b}}(91.27 \mathrm{GeV})}$. 
DELPHI 1994

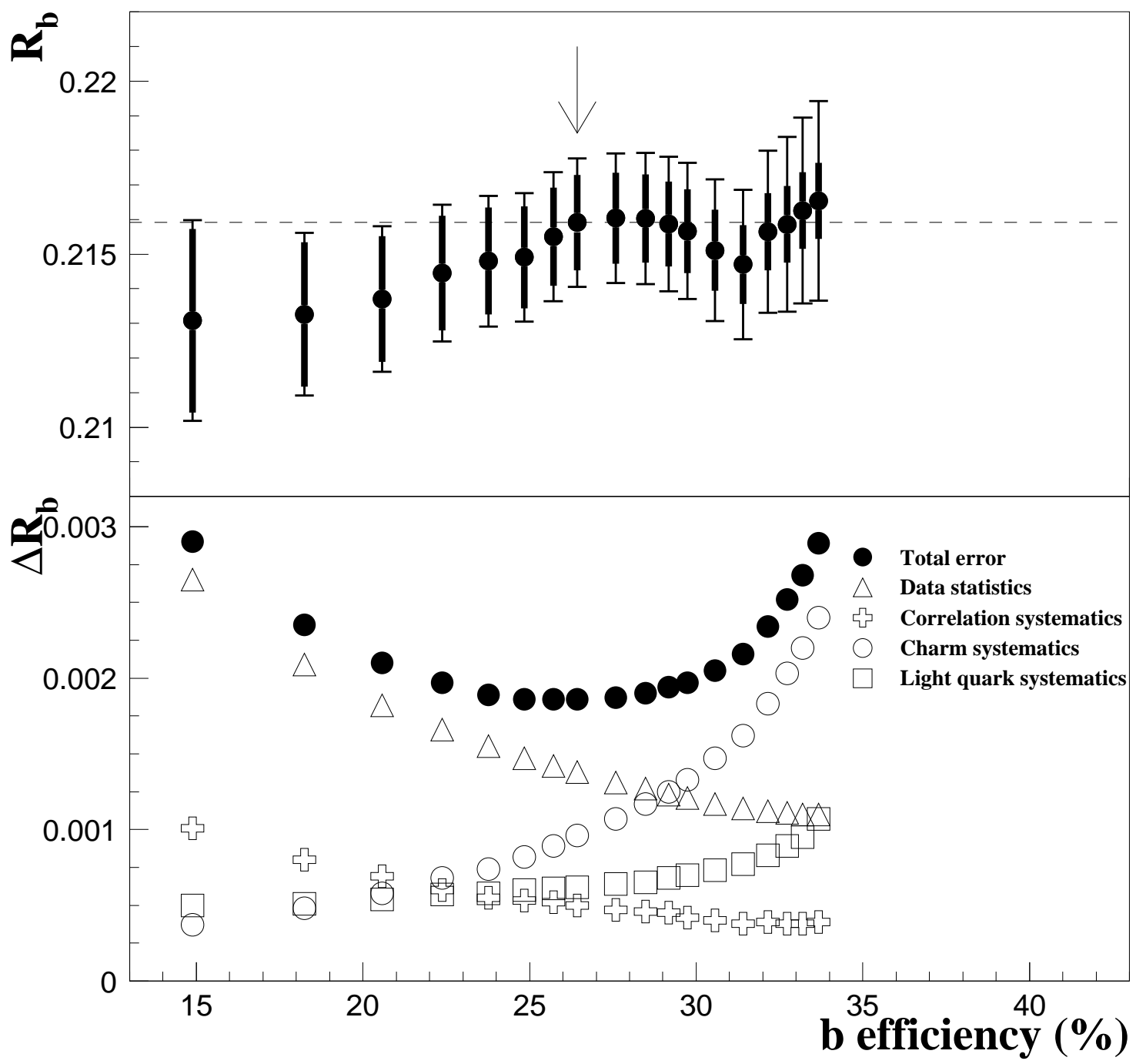

Figure 13: The measured value of $R_{\mathrm{b}}$ from the secondary vertex analysis using the 1994 data set as a function of the b-tagging efficiency taken from the simulation, together with the contributions to the total uncertainty on $R_{\mathrm{b}}$. In the upper plot, the thick error bars show the statistical errors, the thinner bars indicate the total errors, and all errors are correlated from point to point. The arrow marks the position of the cut and the hashed line shows the value at that cut. 


\begin{tabular}{||l|c|c|c|c|c||}
\hline & \multicolumn{5}{|c||}{$\Delta R_{\mathrm{b}} \times 10^{4}$} \\
Error Source & 1992 & 1993 & 1994 & 1995 & Comb. \\
\hline \hline Statistical error & \pm 19.2 & \pm 18.9 & \pm 13.8 & \pm 22.1 & \pm 8.9 \\
\hline Simulation statistics & \pm 8.9 & \pm 6.4 & \pm 4.9 & \pm 9.0 & \pm 3.4 \\
Light quark efficiency & \pm 3.5 & \pm 3.7 & \pm 4.0 & \pm 4.0 & \pm 3.7 \\
Charm quark efficiency & \pm 9.8 & \pm 9.6 & \pm 9.6 & \pm 9.2 & \pm 8.7 \\
Angular correlation & \pm 4.7 & \pm 5.4 & \pm 1.6 & \pm 6.4 & \pm 3.7 \\
Gluon radiation & \pm 3.2 & \pm 3.2 & \pm 3.0 & \pm 3.0 & \pm 3.1 \\
b physics correlation & \pm 0.9 & \pm 1.0 & \pm 0.5 & \pm 0.5 & \pm 0.7 \\
Acceptance bias & \pm 2.3 & \pm 1.8 & \pm 1.3 & \pm 2.2 & \pm 0.9 \\
\hline Total systematic error & \pm 15.0 & \pm 13.8 & \pm 12.1 & \pm 15.4 & \pm 11.2 \\
\hline Total error & \pm 24.4 & \pm 23.4 & \pm 18.3 & \pm 26.9 & \pm 14.3 \\
\hline
\end{tabular}

Table 13: Summary of the systematic uncertainties on $R_{\mathrm{b}}$ from the secondary vertex analysis, for all data sets and for the combined result.

Instead, simply the ratio of the fraction of tagged events can be used, with very small corrections due to changes in the b-tagging efficiency and almost negligible corrections due to background. These corrections were calculated using the simulation. The measurement was performed with the enhanced impact parameter method, but combining the information available from both hemispheres in order to define an event tag. This results in a much higher efficiency for a given purity than hemisphere probabilities. Several different values of the event probability cut were used, and a minimum statistical error was found at a b-purity of $85 \%$. At this value of the cut, the b-tagging efficiency was consistent with being independent of energy within the simulation statistical error of typically $0.2 \%$. It was about $75 \%(81 \%)$ for $1993(1995)$, while the probability to mistag c and uds events was about $11 \%(13 \%)$ and $1.6 \%(1.4 \%)$. The following ratios were found:

$$
\begin{aligned}
& R_{-}=\frac{R_{\mathrm{b}}(89.46 \mathrm{GeV})}{R_{\mathrm{b}}(91.27 \mathrm{GeV})}=0.9909 \pm 0.0081 \\
& R_{+}=\frac{R_{\mathrm{b}}(93.00 \mathrm{GeV})}{R_{\mathrm{b}}(91.27 \mathrm{GeV})}=1.0069 \pm 0.0069 .
\end{aligned}
$$

The error is statistical only. All systematic uncertainties were found to be negligible. The values are consistent with the Standard Model prediction of $0.997(0.998)$ for $R_{-}\left(R_{+}\right)$. Figure 14 shows the stability of the measurement as a function of the b-purity for the two years of data taking.

\section{Comparison with Previous Results}

Using the data taken from 1991 to 1993 DELPHI has published $R_{\mathrm{b}}=0.2213 \pm 0.0016 \pm$ 0.0021 as a combination of several methods. The result with a single/double tag analysis based on impact parameters only was $R_{\mathrm{b}}=0.2219 \pm 0.0018 \pm 0.0028$. The difference with respect to the measurement of this paper is about 2 standard deviations, and similarly when comparing it with the result of only the enhanced impact parameter analysis for 1992-1993 data (see section 4.5).

It is worth noting that the old analysis involved not only a different method (the impact parameter method), but also the data sample was processed with different track pattern recognition, ambiguity processor and track fitting programs. In order to understand the 


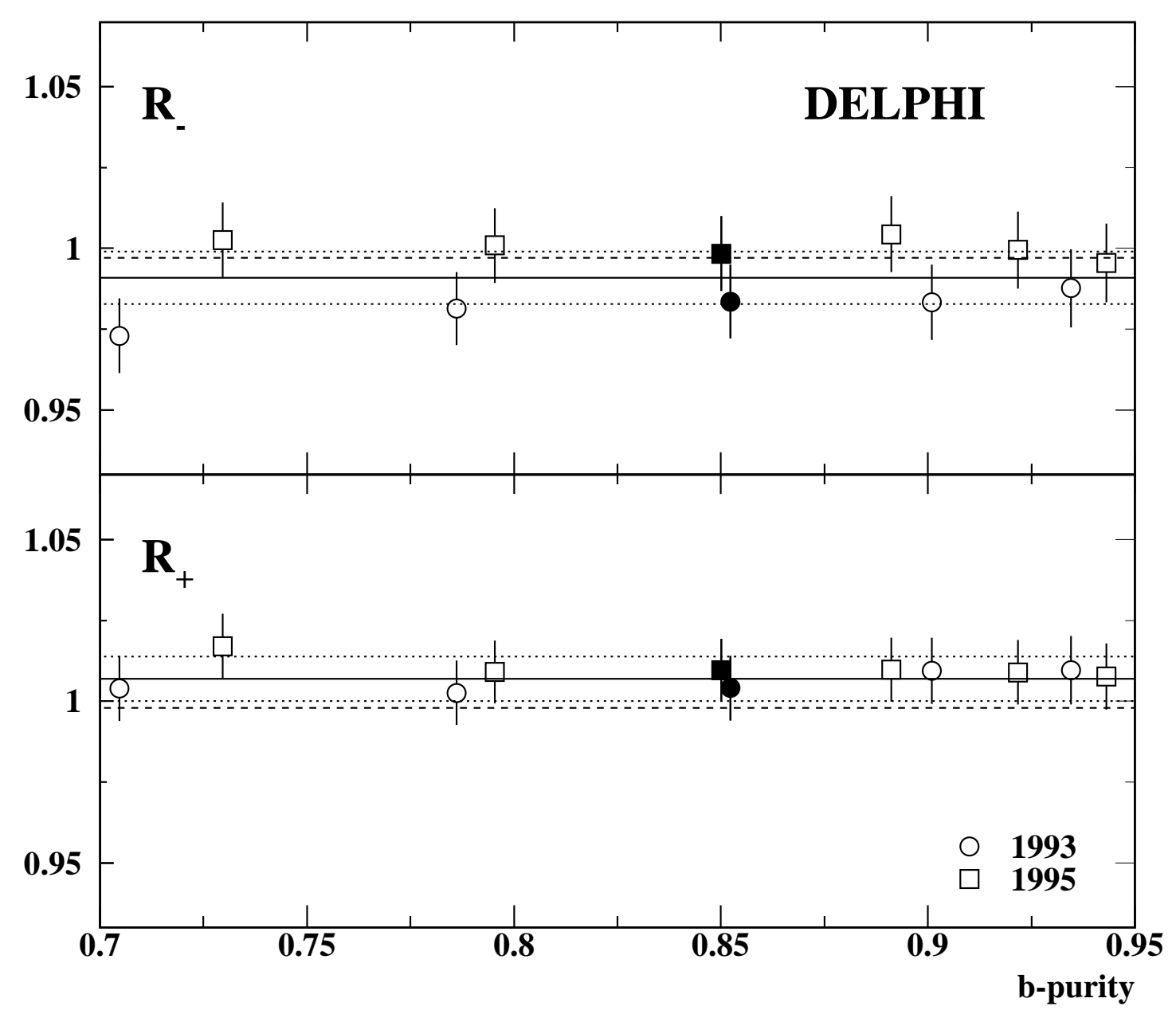

Figure 14: Ratio of the off-peak and on-peak $R_{\mathrm{b}}$ values as a function of the b-purity. The black symbols represent the point of minimal error which have been used for the central result. The solid lines represent the average over the two years with its error indicated by the dotted lines. The dashed lines indicate the Standard Model expectation. 
origin of the discrepancy between the result of this paper and the previous results, two different tests were performed. The analysis of the 1992-1993 data was repeated:

1. with the old data processing using the enhanced impact parameter method;

2. with the new data processing (the one used in this paper) using the "old" impact parameter method.

The following results were obtained:

Test 1: Repeating the analysis of section 4 of this paper with the old data processing and working at a similar value of the purity as used in this paper (but lower b-efficiency, i.e. $\epsilon_{\mathrm{b}} \sim 18 \%$ ), the $R_{\mathrm{b}}$ central value discrepancy is $\sim 1 \%$ (which is compatible within errors), to be compared with $\sim 3 \%$ for the old analysis (old method with old processing). When lowering the purity to get the same background as in the old analysis, the $R_{\mathrm{b}}$ central value rises and the discrepancy is $\sim 2.6 \%$.

Test 2: Repeating the old impact parameter analysis with the reprocessed data and working at the same value of the b-tagging efficiency as in [5] (i.e. for $\epsilon_{\mathrm{b}} \sim 21 \%$ ), the $R_{\mathrm{b}}$ central value is well compatible with the result presented in this paper (i.e. the discrepancy is $\sim 1 \%$ ). With the improved tracking, however, the purity is much higher for this efficiency value. Lowering the cut to get the same charm background, $R_{\mathrm{b}}$ remains stable; however some rise can be seen if the cut is lowered such that the uds-background increases to the same level as in the old analysis.

From these two tests it can be concluded that the new tracking, pattern recognition and ambiguity processor allowed reaching a much higher b-purity for the same b-efficiency. Because of the improved tracking, new tagging methods could be developed. In particular the requirement of a secondary vertex helped in two ways to improve the measurement of $R_{\mathrm{b}}$ compared with that of the previous DELPHI publication: reducing the charm background and the effect of imperfect simulation of pattern recognition mistakes. Wrong associations of vertex detector hits may produce a large impact parameter, but this does not usually result in a fitted secondary vertex.

\section{Results and Conclusions}

Different measurements of the partial decay width $R_{\mathrm{b}}^{0}$ of the $\mathrm{Z}$ into b-hadrons have been performed. Events were selected using tracks having large impact parameters in jets with reconstructed secondary vertices or with a multivariate technique or with a neural network method for secondary vertices.

The enhanced impact parameter analysis compares single and double tag rates to extract $R_{\mathrm{b}}$, using a highly efficient and pure b-tag. The enhanced impact parameter tag of this analysis is then used as the primary b-tag in the multivariate analysis, which includes additional tags for b-, c- and light quarks. The information of the first analysis is therefore fully included in the second one and no further combination is possible.

The statistical correlation between the multivariate and the secondary vertex analyses has been evaluated to be small and the results are thus statistically consistent. However, due to the large systematic correlation, no improvement can be obtained by a combination, so that the secondary vertex analysis is used as a cross-check. An additional cross-check has been performed with the b-confidence tag [23].

Using about 3.4 million hadronic $\mathrm{Z}$ events collected in the years 1992 to 1995 by DELPHI and combining all centre-of-mass energies at which LEP has run, the following results were obtained: 


\begin{tabular}{|c|c|c|}
\hline Source of error & Range & $\Delta R_{\mathrm{b}} \times 10^{4}$ \\
\hline Data statistics & & \pm 6.7 \\
\hline Simulation statistics & & \pm 3.3 \\
\hline Event selection & & \pm 0.9 \\
\hline Tracking & & \pm 1.3 \\
\hline $\mathrm{K}^{0}, \Lambda^{0}$, photons, etc. & see text & $\mp 0.4$ \\
\hline Gluon splitting $g \rightarrow c \bar{c}$ & $(2.33 \pm 0.50) \%$ & $\mp 0.8$ \\
\hline Gluon splitting $g \rightarrow \mathrm{b} \overline{\mathrm{b}}$ & $(0.269 \pm 0.067) \%$ & $\mp 2.7$ \\
\hline $\mathrm{D}^{+}$fraction in $\mathrm{c} \overline{\mathrm{c}}$ events & $0.233 \pm 0.027$ & $\mp 1.2$ \\
\hline $\mathrm{D}_{\mathrm{s}}$ fraction in $\mathrm{c} \overline{\mathrm{c}}$ events & $0.103 \pm 0.029$ & \pm 0.3 \\
\hline c-baryon fraction in $c \bar{c}$ events & $0.063 \pm 0.028$ & \pm 1.2 \\
\hline $\mathrm{BR}\left(\mathrm{D}^{0} \rightarrow\right.$ no neutrals $)$ & $(14.1 \pm 1.1) \%$ & $\mp 0.6$ \\
\hline $\mathrm{BR}\left(\mathrm{D}^{0} \rightarrow 1\right.$ neut., $\geq 2$ charged $)$ & $(37.7 \pm 1.7) \%$ & $\mp 0.3$ \\
\hline $\mathrm{BR}\left(\mathrm{D}^{+} \rightarrow\right.$ no neutrals $)$ & $(11.2 \pm 0.6) \%$ & $\mp 0.5$ \\
\hline $\mathrm{BR}\left(\mathrm{D}^{+} \rightarrow 1\right.$ neut.,$\geq 2$ charged $)$ & $(26.1 \pm 2.3) \%$ & $\mp 0.2$ \\
\hline $\mathrm{BR}\left(\mathrm{D}_{\mathrm{s}} \rightarrow K^{0} X\right)$ & $(33 \pm 18) \%$ & \pm 1.2 \\
\hline $\mathrm{D}^{0}$ lifetime & $0.415 \pm 0.004 \mathrm{ps}$ & $\mp 0.3$ \\
\hline $\mathrm{D}^{+}$lifetime & $1.057 \pm 0.015 \mathrm{ps}$ & $\mp 0.3$ \\
\hline $\mathrm{D}_{\mathrm{s}}$ lifetime & $0.447 \pm 0.017 \mathrm{ps}$ & $\mp 0.3$ \\
\hline$\Lambda_{\mathrm{c}}$ lifetime & $0.206 \pm 0.012 \mathrm{ps}$ & $\mp 0.0$ \\
\hline D decay multiplicity & see $[18]$ & $\mp 0.8$ \\
\hline$\left\langle x_{E}(\mathrm{c})\right\rangle$ & $0.484 \pm 0.008$ & $\mp 0.5$ \\
\hline Two b's same hemisphere & $\pm 30 \%$ & \pm 0.5 \\
\hline$\left\langle x_{E}(\mathrm{~b})\right\rangle$ & $0.702 \pm 0.008$ & \pm 1.2 \\
\hline B decay multiplicity & $4.97 \pm 0.07$ & $\mp 0.9$ \\
\hline Average B lifetime & $1.55 \pm 0.05 \mathrm{ps}$ & $\mp 0.0$ \\
\hline Angular effects & see text & \pm 0.9 \\
\hline Gluon radiation & see text & \pm 2.2 \\
\hline \multicolumn{2}{|l|}{ Total systematic error } & \pm 6.0 \\
\hline \multicolumn{2}{|l|}{ Total error } & \pm 9.0 \\
\hline
\end{tabular}

Table 14: Detailed error breakdown for the measurement of $R_{\mathrm{b}}$ from the multivariate analysis for the combined result.

with the enhanced impact parameter analysis:

$$
R_{\mathrm{b}}=0.21668 \pm 0.00088(\text { stat }) \pm 0.00070(\text { syst })-0.024\left(R_{\mathrm{c}}-0.172\right),
$$

with the secondary vertex analysis:

$$
R_{\mathrm{b}}=0.21722 \pm 0.00089(\text { stat }) \pm 0.00112(\text { syst })-0.088\left(R_{\mathrm{c}}-0.172\right),
$$

with the b-confidence tag:

$$
R_{\mathrm{b}}=0.21855 \pm 0.00072 \text { (stat) } \pm 0.00134(\text { syst })-0.068\left(R_{\mathrm{c}}-0.172\right),
$$

and with the multivariate analysis:

$$
R_{\mathrm{b}}=0.21616 \pm 0.00067(\text { stat }) \pm 0.00060(\text { syst })-0.024\left(R_{\mathrm{c}}-0.172\right) .
$$


Taking common errors into account the agreement between (25) and (23) is within one standard deviation and between (25) and (24) within 1.5 standard deviations. The agreement between (25) and (22) can be judged from the good fit $\chi^{2}$ given in section 5.3.

Due to the large systematic correlation no gain can be obtained from a combination, so that the result with the smallest error, coming from the multivariate analysis, is used as the final result. Applying the small $(+0.00018)$ correction for photon exchange thus yields for the ratio of partial widths:

$$
R_{\mathrm{b}}^{0}=0.21634 \pm 0.00067(\text { stat }) \pm 0.00060(\text { syst })-0.024\left(R_{\mathrm{c}}-0.172\right) .
$$

The detailed breakdown of the error for this measurement is given in table 14 .

The result supersedes the previous DELPHI result [5] and is in agreement with those of other measurements at LEP and SLC [3,4,6-8], improving the precision significantly. It is also in good agreement with the Standard Model expectation of $R_{\mathrm{b}}^{0}=0.21584 \mp 0.00018$ [27], assuming a mass of the top quark of $m_{t}=173.8 \pm 5.2 \mathrm{GeV} / c^{2}[9]$.

In addition the energy dependence of $R_{\mathrm{b}}$ around the $\mathrm{Z}$ peak has been measured, yielding:

$$
\begin{aligned}
& R_{-}=\frac{R_{\mathrm{b}}(89.46 \mathrm{GeV})}{R_{\mathrm{b}}(91.27 \mathrm{GeV})}=0.9909 \pm 0.0081, \\
& R_{+}=\frac{R_{\mathrm{b}}(93.00 \mathrm{GeV})}{R_{\mathrm{b}}(91.27 \mathrm{GeV})}=1.0069 \pm 0.0069,
\end{aligned}
$$

which is also consistent with the Standard Model prediction. 


\section{Acknowledgements}

We are greatly indebted to our technical collaborators, to the members of the CERNSL Division for the excellent performance of the LEP collider, and to the funding agencies for their support in building and operating the DELPHI detector.

We acknowledge in particular the support of Austrian Federal Ministry of Science and Traffics, GZ 616.364/2-III/2a/98, FNRS-FWO, Belgium,

FINEP, CNPq, CAPES, FUJB and FAPERJ, Brazil, Czech Ministry of Industry and Trade, GA CR 202/96/0450 and GA AVCR A1010521, Danish Natural Research Council,

Commission of the European Communities (DG XII),

Direction des Sciences de la Matière, CEA, France,

Bundesministerium für Bildung, Wissenschaft, Forschung und Technologie, Germany,

General Secretariat for Research and Technology, Greece,

National Science Foundation (NWO) and Foundation for Research on Matter (FOM), The Netherlands,

Norwegian Research Council,

State Committee for Scientific Research, Poland, 2P03B06015, 2P03B03311 and SPUB/P03/178/98,

JNICT-Junta Nacional de Investigação Científica e Tecnológica, Portugal,

Vedecka grantova agentura MS SR, Slovakia, Nr. 95/5195/134,

Ministry of Science and Technology of the Republic of Slovenia,

CICYT, Spain, AEN96-1661 and AEN96-1681,

The Swedish Natural Science Research Council,

Particle Physics and Astronomy Research Council, UK,

Department of Energy, USA, DE-FG02-94ER40817. 


\section{References}

[1] The LEP Collaborations and SLD, A Combination of Preliminary Electroweak Measurements and Constraints on the Standard Model, CERN-PPE/97-154.

[2] J. Ellis, J. L. Lopez and D. V. Nanopoulos, Phys. Lett. B397 (1997) 88.

[3] ALEPH Collaboration, R. Barate et al., Phys. Lett.B401 (1997) 150.

[4] ALEPH Collaboration., R. Barate et al., Phys. Lett. B401 (1997) 163.

[5] DELPHI Collaboration, P. Abreu et al., Z. Phys. C70 (1996) 531. DELPHI Collaboration, P. Abreu et al., Z. Phys. C66 (1995) 323.

[6] L3 Collaboration, O. Adriani et al., Phys. Lett. B307 (1993) 237.

[7] OPAL Collaboration, G. Abbiendi et al., CERN-EP/98-137 (1998)

[8] SLD Collaboration, K. Abe et al., Phys. Rev. Lett. 80 (1998) 660.

[9] The Particle Data Group, C. Caso et al., E. Phys. J. C3 (1998) 1.

[10] DELPHI Collaboration, P. Aarnio et al., Nucl. Inst. Meth. A303 (1991) 233.

[11] DELPHI Collaboration, P. Abreu et al., Nucl. Inst. Meth. A378 (1996) 57.

[12] N. Bingefors et al., Nucl. Inst. Meth. A328 (1993) 447.

[13] V. Chabaud et al., Nucl. Inst. Meth A368 (1996) 314.

[14] T. Sjöstrand et al., in "Z physics at LEP 1", CERN 89-08, CERN, Geneva, 1989; Comp. Phys. Comm. 39 (1986) 347.

[15] DELPHI Collaboration, P. Abreu at al., Z. Phys. C77 (1996) 11.

[16] G. Borisov and C. Mariotti, Nucl. Inst. Meth. A372 (1996) 181.

[17] ALEPH Collaboration, D. Buskulic et al., Phys. Lett. B313 (1993), 535.

[18] The LEP Collaborations, Nucl. Inst. Meth. A378 (1996) 101; The LEP Heavy Flavour Working Group, Presentation of LEP Electroweak Heavy Flavour Result for Summer 1996 Conferences, LEPHF 96-01; The LEP Heavy Flavour Working Group, Input parameters for the LEP electroweak Heavy Flavour Results for Summer 1998 Conferences, LEPHF/98-01.

[19] DELPHI Collaboration, P. Abreu et al., Phys. Lett. B405 (1997) 202, ALEPH Collaboration, R. Barate et al., A measurement of the Gluon Splitting Rate into $b \bar{b}$ Pairs in Hadronic Z decays, CERN-EP/98-103 (submitted to Phys. Lett. B).

[20] DELPHI Collaboration, P. Abreu et al., Phys. Lett. B425 (1998) 399.

[21] P. Billoir et al., Nucl. Inst. Meth. A360 (1995) 532.

[22] Ch. De la Vaissiere, F. Martinez-Vidal, Description and performances of the DELPHI multivariate flavour tagging, internal DELPHI note, DELPHI 97-134 PHYS 721.

[23] T. Hessing, Measurement of $R_{b}$ using the Improved Flavour Confidence Method, internal DELPHI Note, DELPHI 98-151 PHYS 795.

[24] A. Zell et al., Stuttgart Neural Network Simulator, University of Stuttgart, Institute for Parallel and Distributed High Performance Systems.

[25] F. Caravaglios and G. Ross, Phys. Lett. B346 (1995) 159.

[26] J. Erler, J. L. Feng and N. Polonsky, Phys. Rev. Lett. 78 (1997) 3063.

[27] D. Bardine et al., ZFITTER: An Analytical Program for Fermion Pair Production in $e^{+} e^{-}$Annihilation, CERN-TH 6443/92 (May 1992). 